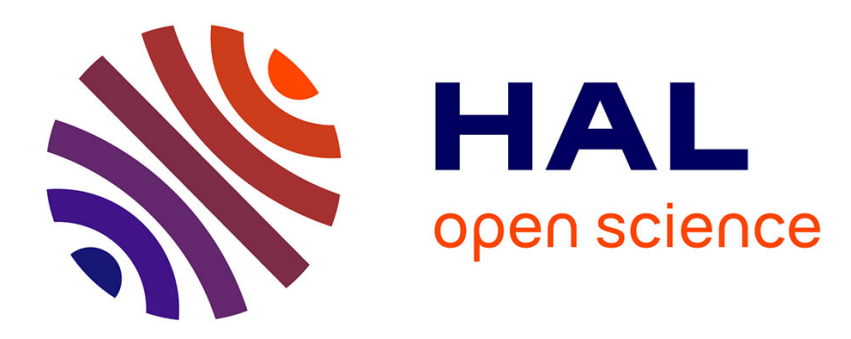

\title{
Compositions of states and observables in Fock spaces
}

L. Amour, L. Jager, J. Nourrigat

\section{To cite this version:}

L. Amour, L. Jager, J. Nourrigat. Compositions of states and observables in Fock spaces. Reviews in Mathematical Physics, 2020, 32 (05), pp.2050012. 10.1142/S0129055X20500129 . hal-03477567

\section{HAL Id: hal-03477567 https://hal.science/hal-03477567}

Submitted on 13 Dec 2021

HAL is a multi-disciplinary open access archive for the deposit and dissemination of scientific research documents, whether they are published or not. The documents may come from teaching and research institutions in France or abroad, or from public or private research centers.
L'archive ouverte pluridisciplinaire HAL, est destinée au dépôt et à la diffusion de documents scientifiques de niveau recherche, publiés ou non, émanant des établissements d'enseignement et de recherche français ou étrangers, des laboratoires publics ou privés. 


\title{
Compositions of states and observables in Fock spaces
}

\author{
L. Amour, L. Jager, J. Nourrigat \\ Université de Reims, France
}

\begin{abstract}
This article is concerned with compositions in the context of three standard quantizations in the Fock spaces framework, namely, anti-Wick, Wick and Weyl quantizations. The first one is a composition of states also known as a Wick product and is closely related to the standard scattering identification operator encountered in Quantum Electrodynamics for time dynamics issues (see [29, 13]). Anti-Wick quantization and Segal-Bargmann transforms are implied here for that purpose. The other compositions are for observables (operators in some specific classes) for the Wick and Weyl symbols. For the Wick and Weyl symbols of the composition of two operators, we obtain an absolutely converging series and for the Weyl symbol, the remainder terms up to any orders of the expansion are controlled, still in the Fock space framework.
\end{abstract}

Keywords: Scattering identification operator, composition, quantization, Fock spaces, infinite dimensional analysis, composition of states, Wick product, Wick composition, Weyl composition, composition of operators, anti-Wick quantization, Wick quantization, Wick symbol, Husimi function, Weyl symbol, Mizrahi series, Wiener spaces, Segal-Bargmann transform, heat operator, symbolic calculus, semiclassical analysis, QED, quantum electrodynamics.

MSC 2010: 35S05, 47G30, 81Q20, 47L80, 26E15, 28C20.

\section{Contents}

1 Introduction and main results. $\quad 1$

1.1 Some general tools in Fock spaces. . . . . . . . . . . . . . . . . . . . . . 2

1.2 Quantizations in Fock spaces: examples. . . . . . . . . . . . . . . . . . 5

1.3 Wick and Weyl symbols for composition of operators. . . . . . . . . . . . . . . . . 10

2 Segal Bargmann transformation and Wick product. 12

2.1 Segal isomorphism. . . . . . . . . . . . . . . . . . . . . . . . 12

2.2 Segal Bargmann transform. . . . . . . . . . . . . . . . . . . . . . . . . . 13

2.3 Power series expansions. . . . . . . . . . . . . . . . . . . . . . 15

2.4 Connection between $T_{h}^{F H} f$ and $T_{h}^{F W} f \ldots \ldots \ldots \ldots \ldots \ldots$. . . . . . . . . . . . . 18

2.5 Segal Bargmann transform and Wick product: proof of Proposition ?? and Theorem ?? . 19

3 Mizrahi series: proof of Theorem ??. 21

4 Weyl symbol composition: proof of Theorem ??. $\quad 22$ 


\section{Introduction and main results.}

The aim of this article is to provide additional details and to make some clarifications on standard and commonly used tools when working in Fock spaces, especially for the purpose of studying spin boson type models.

In order to be more specific concerning the model we usually consider for applications, let us mention that we are particularly interested in a model that can be used in the analysis of Nuclear Magnetic Resonance (NMR) in the framework of Quantum Electrodynamics (QED). This model is a spin boson type model and can be called spin photon or NMR model. The Hamiltonian is given by F. A. Reuse in [47]. Thus, the Hamiltonian is closely related to the standard spin boson model as a mathematical tool and its interest is justified by the importance of RMN in physics. We also underline that, as a model of QED, this Hamiltonian is much easier to study than the usual Pauli-Fierz Hamiltonian. For a precise definition and for various results of the NMR model, see $[4,5,6]$ which often rely on the pseudodifferential calculus in Fock spaces studied in [3].

We next turn to Fock space setting. The first Subsection below is devoted to some general tools in Fock spaces. The second and third Subsections particularly focus on quantization in Fock spaces.

\subsection{Some general tools in Fock spaces.}

Before considering quantizations in the next two Subsections 1.2 and 1.3, we give some further precisions, results and remarks on general tools which in principle are standard facts (see, e.g., [1, 18, 41, 50, 52, 8, $32])$.

Recall that Fock spaces and tensor products are very often used in the framework of infinite dimensional analysis for Quantum Field Theory (QFT) issues, see e.g., [48, 17] and see also [50, 46].

For any infinite dimensional real separable Hilbert space $H, \mathcal{F}_{s}\left(H_{\mathbf{C}}\right)$ denotes the symmetrized Fock space over $H_{\mathbf{C}}$ where $H_{\mathbf{C}}$ stands for $H+i H$ the standard complexification of $H$ (see [45]). With the usual notations ([45]), we have the decomposition:

$$
\mathcal{F}_{s}\left(H_{\mathbf{C}}\right)=\bigoplus_{m \geq 0} H^{(m)} .
$$

Recall that $H^{(m)}$ is the Hilbert tensor product (while $E^{m}$ is the completed direct sum when $E$ is a Banach space). For all $X$ in $H^{2}, a(X), a^{\star}(X)$ and $\Phi_{S}(X)$ denote annihilation and creation operators, and Segal fields, which are unbounded operators in $\mathcal{F}_{s}\left(H_{\mathbf{C}}\right)$, associated with $X$ (see [46]). The state $\Psi_{0}$ belonging to $\mathcal{F}_{s}\left(H_{\mathbf{C}}\right)$ stands for the vacuum state. The subspace $\mathcal{F}_{s}^{\text {fin }}\left(H_{\mathbf{C}}\right)$ refers here to the set of all finite linear combinations of elements belonging to a standard basis of $\mathcal{F}_{s}\left(H_{\mathbf{C}}\right)$ recalled in $(2.1)$. The creation and annihilation operators in Fock spaces are also related to the derivative operator $D$ in the Malliavin calculus [39], see [44] and see also [1] in the case of manifolds. Also, the creation and annihilation operators $a_{t}$ and $a_{t}^{*}$ correspond to $\partial_{t}$ and its adjoint $\partial_{t}^{*}$ defined using the white noise functional derivative, in the 1-dimensional time parameter context, see [34, 35] and also [25].

A first tool related to Fock spaces is the Gaussian measure setting, see e.g., [20, 36], see also [52, 50, 51, $18,9]$. This setting is also often considered for QFT issues. The first step is to associate a measure space 
$B$ with the Hilbert space $H$ under consideration. Let us recall a standard procedure for the definition of measure spaces $B$ starting from a real separable Hilbert space $H$.

Theorem 1.1. (Gross [19]-[22], Kuo [36]). Let $H$ be a real separable Hilbert space. Then, there exists a (non unique) Banach space $B$ containing $H$, such that $B^{\prime} \subset H^{\prime}=H \subset B$, each space being dense in the following, and for all $h>0$, there exists a probability measure $\mu_{B, h}$ on the Borel $\sigma$-algebra of $B$ with the two following properties:

i) For each $a \in B^{\prime} \subset H$, denoting by $|a|$ the norm of $a$ in $H$ and by $a(x)$ the duality between $B^{\prime}$ and $B$, we have:

$$
\int_{B} e^{i a(x)} d \mu_{B, h}(x)=e^{-\frac{h}{2}|a|^{2}}, \quad a \in B^{\prime} .
$$

ii) For each finite dimensional subspace $E$ of $H$, there is an isometric inclusion $i_{E}$ of $L^{p}\left(E, \mu_{E, h}\right)$ in $L^{p}\left(B, \mu_{B, h}\right),(1 \leq p<\infty)$ where $\mu_{E, h}$ refers to Gaussian measures of variance $h$.

The exact conditions to be fulfilled by $B$ together with the properties involved in this paper are recalled in [3]. In particular, the isometric inclusion $i_{E}$ can be used in order to extend the orthogonal projection $\pi_{E}: H \rightarrow E$ to a $\mu_{B, h}$-almost everywhere defined mapping $\tilde{\pi}_{E}: B \rightarrow E$. For any orthonormal basis $\left(u_{n}\right)_{1 \leq n \leq \operatorname{dim} E}$ the projection is given by $\pi_{E} u=\sum_{n=1}^{\operatorname{dim} E}\left\langle u, u_{n}\right\rangle u_{n}$. By using $\ell_{u_{n}}=i_{E}\left(\left\langle\cdot, u_{n}\right\rangle\right)$ the mapping $\tilde{\pi}_{E}$ defined as $\tilde{\pi}_{E}(x)=\sum_{n=1}^{\operatorname{dim} E} \ell_{u_{n}}(x) u_{n}$ does not depend on the orthonormal basis $\left(u_{n}\right)_{1 \leq n \leq \operatorname{dim} E}$. The gaussian measure $\mu_{E}$ is nothing but the image measure of $\mu_{B, h}$ by $\tilde{\pi}_{E}$ and for any $\mu_{E}-$ measurable function $f$ on $E, f \circ \tilde{\pi}_{E}$ is a $\mu_{B, h}$-measurable function which coincides with $i_{E} f$ if $f \in L^{p}\left(E, \mu_{E, h}\right)$.

In the following, we say that $B$ is a Wiener extension of $H$. Then, one notes that $B^{2}$ is also a Wiener extension of $H^{2}$.

There are other ways for defining $B$. In the case $H=L^{2}\left(\mathbb{R}^{d}\right)$, the space $B=\mathcal{S}^{\prime}\left(\mathbb{R}^{d}\right)$ equipped with a Gaussian measure is often used $([50])$ and its elements are related to white noises $([25,26])$, and other cases as $H$ being the Sobolev spaces of order -1 or $-\frac{1}{2}$ are classical in quantum field theory $([18,50])$, using Minlos Theorem. The Hilbert space $H$ being a Sobolev space of order 1 in one dimension with a vanishing boundary condition is related to the Cameron-Martin space $([12,44])$. One can also get $B=C([0,1])$ the classical Wiener space $([36])$. Configuration spaces taken as Riemannian manifolds can also be considered in this point of view with Poisson measures [1].

The Segal isomorphism $S_{h}$ between the Fock space $\mathcal{F}_{s}\left(H_{\mathbf{C}}\right)$ and the square integrable random variable space $L^{2}\left(B, \mu_{B, h}\right)$ is the very classical second tool and is recalled in Section 2.1.

The third tool is the Segal Bargmann transform. In the finite dimensional framework, the Segal Bargmann transform of a function $f \in L^{2}\left(\mathbb{R}^{n}\right)$ is a function belonging to $L^{2}\left(\mathbb{R}^{2 n}\right)$ (with suitable measures) and holomorphic when $\mathbb{R}^{2 n}$ is identified with $\mathbf{C}^{n}$ (see [15]).

In our case, $f$ is an element of $\mathcal{F}_{s}\left(H_{\mathbf{C}}\right)$. Yet the Segal Bargmann transform of $f$ is not both holomorphic and belonging to a $L^{2}$ space and we have to therefore define two Segal Bargmann transforms. One Segal Bargmann transform of $f$ is denoted by $T_{h}^{F H} f$. It is a function defined on $H^{2}$ and Gateaux antiholomorphic if $H^{2}$ is identified with $H_{\mathbf{C}}$ (the anti-holomorphy instead of holomorphy comes from our notations). Concerning the other Segal Bargmann transform of $f$, the simplest way is to defined it as an element denoted $T_{h}^{F F} f$ belonging to the Fock space $\mathcal{F}_{s}\left(H_{\mathbf{C}} \times H_{\mathbf{C}}\right)$. It can also be viewed as an element $T_{h}^{F W} f$ belonging to a $L^{2}$ space via the Segal isomorphism. The fact that the function $T_{h}^{F H} f$ (defined in 
(2.4)) is Gateaux anti-holomorphic identifying $H^{2}$ with $H_{\mathbf{C}}$ is a well known property (see [24, 14]). We also recover this fact in point $i i)$ of Proposition 2.5. The $H$ notation refers to anti-Holomorphic, $W$ to Wiener stochastic extension and $F$ to Fock space representation.

This last property then raises the issue of entire power series expansion of $T_{h}^{F H} f$ (with monomials defined in (2.9)). The sense of convergence of this expansion is specified in Proposition 2.5. We emphasize that it is not an absolutely convergent series. We then have defined two functions $T_{h}^{F H} f$ and $T_{h}^{F W} f$ for any $f \in \mathcal{F}_{s}\left(H_{\mathbf{C}}\right)$, one function being defined on $H^{2}$ whereas the other one is defined almost everywhere on $B^{2}$. Proposition 2.8 makes the link between these two functions. In particular, the second function is the stochastic extension (in the sense of Definition 1.6) of the first one, while the the first function is the reproducing kernel applied to the second one.

These Segal Bargmann transforms in infinite dimension are defined and studied in Subsections 2.2-2.4. One can also see, e.g., [41] in a probabilistic point of view and [8] in a more mathematical physics context.

We now turn to Wick products and the scattering identification operator. In [29], [54] (formula (17.92)), $[13,16]$, a composition law is introduced in $\mathcal{F}_{s}^{\text {fin }}\left(H_{\mathbf{C}}\right)$ in order to construct a scattering identification operator for some spin boson models (see also Section 1.2). This composition law is defined in the following way.

Definition 1.2. With the above notations, one denotes by $I$ the bilinear map from $\mathcal{F}_{s}^{\text {fin }}\left(H_{\mathbf{C}}\right) \times \mathcal{F}_{s}^{\text {fin }}\left(H_{\mathbf{C}}\right)$ to $\mathcal{F}_{s}\left(H_{\mathbf{C}}\right)$ satisfying for all $X_{1}, \ldots, X_{m}$ and all $Y_{1}, \ldots Y_{p}$ in $H$ :

$$
I\left(a^{\star}\left(X_{1}\right) \ldots a^{\star}\left(X_{m}\right) \Psi_{0}, a^{\star}\left(Y_{1}\right) \ldots a^{\star}\left(Y_{p}\right) \Psi_{0}\right)=a^{\star}\left(X_{1}\right) \ldots a^{\star}\left(X_{m}\right) a^{\star}\left(Y_{1}\right) \ldots a^{\star}\left(Y_{p}\right) \Psi_{0} .
$$

Also recall that the Wick product is the composition law usually defined in $L^{2}\left(B, \mu_{B, h}\right)$ as follows. Let $f$ and $g$ be the images under the Segal isomorphism $S_{h}$ respectively of two elements in the subspaces $H^{(m)}$ and $H^{(n)}$ of the direct sum decomposition (1.1). Then the Wick product denoted by : $f, g:$ (or also : $f g$ : or $f: g$ ) is the orthogonal projection of the ordinary product $f(\cdot) g(\cdot)$ on the image of $H^{(m+n)}$ under $S_{h}$ (see [32]). We remind that the Wick product can also be defined by iteration still in the random variable setting (see [50]).

Proposition 1.3. Fix $h>0$. With these notations, one has:

$$
: S_{h} f, S_{h} g:=S_{h} I(f, g)
$$

for any $f$ and $g$ in $\mathcal{F}_{s}^{\text {fin }}\left(H_{\mathbf{C}}\right)$.

Proof of Proposition 1.3. We use the standard Hilbertian basis $\left(u_{\alpha}\right)$ recalled in $(2.1)$. On one side, it is checked in (2.21) that,

$$
I\left(u_{\alpha}, u_{\beta}\right)=\left(\frac{(\alpha+\beta) !}{\alpha ! \beta !}\right)^{1 / 2} u_{\alpha+\beta}
$$

and on the other side, with $S_{h} u_{\alpha}$ defined in (2.2), it is well known that (see for example Example 3.48 of [32]),

$$
: S_{h} u_{\alpha}, S_{h} u_{\beta}:=\left(\frac{(\alpha+\beta) !}{\alpha ! \beta !}\right)^{1 / 2} S_{h} u_{\alpha+\beta}
$$


The proposition then follows.

Next, we extend in Proposition 1.4 below the domain of definition of the law $I$, or equivalently of the Wick product.

For any $R \geq 1$, denote by $\mathcal{F}_{s}^{R}\left(H_{\mathbf{C}}\right)$ the subspace of all $f=\left(f_{n}\right)$ in $\mathcal{F}_{s}\left(H_{\mathbf{C}}\right)$ such that the following series is converging:

$$
\|f\|_{R}^{2}=\sum_{n \geq 0} R^{n}\left\|f_{n}\right\|^{2}<\infty .
$$

In particular, $\mathcal{F}_{s}^{1}\left(H_{\mathbf{C}}\right)=\mathcal{F}_{s}\left(H_{\mathbf{C}}\right)$.

Proposition 1.4. Fix $R \geq 1, R^{\prime} \geq 1, R^{\prime \prime} \geq 1$ satisfying $\frac{1}{R^{\prime \prime}}=\frac{1}{R}+\frac{1}{R^{\prime}}$. Then, the composition law $I$ in Definition 1.2 is extended to a continuous bilinear map from $\mathcal{F}_{s}^{R}\left(H_{\mathbf{C}}\right) \times \mathcal{F}_{s}^{R^{\prime}}\left(H_{\mathbf{C}}\right)$ into $\mathcal{F}_{s}^{R^{\prime \prime}}\left(H_{\mathbf{C}}\right)$. For all $f$ in $\mathcal{F}_{s}^{R}\left(H_{\mathbf{C}}\right)$ and $g$ in $\mathcal{F}_{s}^{R^{\prime}}\left(H_{\mathbf{C}}\right)$, we have:

$$
\|I(f, g)\|_{R^{\prime \prime}} \leq\|f\|_{R}\|g\|_{R^{\prime}}
$$

Proposition 1.4 is proved in Section 2.5. We also study the connection between this composition law $I$ and the Segal Bargmann transform in Section 2.5 by proving the following result which is related to some results of $[42,40]$.

Theorem 1.5. i) Fix $h>0$. Let $R \geq 1, R^{\prime} \geq 1, R^{\prime \prime} \geq 1$ satisfying $\frac{1}{R^{\prime \prime}}=\frac{1}{R}+\frac{1}{R^{\prime}}$. Then,

$$
T_{h}^{F H} I(f, g)=\left(T_{h}^{F H} f\right)\left(T_{h}^{F H} g\right)
$$

for any $f \in \mathcal{F}_{s}^{R}\left(H_{\mathbf{C}}\right)$ and $g \in \mathcal{F}_{s}^{R^{\prime}}\left(H_{\mathbf{C}}\right)$ where the composition in the right hand side refers to the ordinary product of functions defined on $H^{2}$.

ii) Let $h>0$. Fix any Wiener extension $B$ of a real separable Hilbert space H. Then,

$$
T_{h}^{F W} I(f, g)(X)=\left(T_{h}^{F W} f\right)(X)\left(T_{h}^{F W} g\right)(X),
$$

for almost every $X \in B^{2}$.

Therefore, via Segal Bargmann transform, one notes that the composition law $I$ is reduced to the usual pointwise multiplication for functions.

A last property satisfied by the composition $I$ (or Wick product) concerns the Wick product of two coherent states. The definition of coherent states $\Psi_{X, h}$ in recalled in (1.15) for any $X \in H^{2}$ and $h>0$. A direct computation also shows that:

$$
\Psi_{X, h}=\sum_{m \geq 0} \frac{e^{-\frac{|q|^{2}+|p|^{2}}{4 h}}}{(2 h)^{\frac{m}{2}}(m !)^{\frac{1}{2}}}(q+i p) \otimes \cdots \otimes(q+i p),
$$

where $X=(q, p)$ and $h>0$. Equality (1.9) shows in particular that $\Psi_{X, h} \in \mathcal{F}_{s}^{R}\left(H_{\mathbf{C}}\right)$ for each $R>1$. This implies that the Wick product $I\left(\Psi_{X, h}, \Psi_{Y, h}\right)$ of the two coherent states $\Psi_{X, h}$ and $\Psi_{Y, h}$ where $X \in H^{2}$ and $Y \in H^{2}$ is well defined. We then prove the following equality in Section 2.5:

$$
I\left(\Psi_{X, h}, \Psi_{Y, h}\right)=e^{\frac{1}{2 h} X \cdot Y} \Psi_{X+Y, h},
$$

for any $X$ and $Y$ in $H^{2}$, and $h>0$. 


\subsection{Quantizations in Fock spaces: examples.}

Before stating in the next Subsection 1.3 two results concerning Wick and Weyl quantizations, let us here remind quantization definitions and give some applications to spin boson type models which are our main objective.

Fix a real separable Hilbert space $H$. There are at least three ways to make a relation between a function $F$ on $H^{2}$ and an operator: the anti-Wick, Weyl and Wick quantizations.

Anti-Wick quantization. We first note, in the aim to define an anti-Wick operator on $\mathcal{F}_{s}\left(H_{\mathbb{C}}\right)$ from a (symbol) function $F$ defined on $H^{2}$ and in contrast to the finite dimension case, that it is not sufficient for a symbol to be a bounded continuous function. Indeed, we assume in addition that the symbol has a stochastic extension in sense of the following definition.

Definition 1.6. Let $H$ be a separable real Hilbert space of infinite dimension. Set $B$ a Wiener extension of $H$ (see Theorem 1.1) and set $\mu_{B, h}$ the gaussian measure with variance $h$. One says that a continuous function $F$ on $H$ admits a stochastic extension in $L^{p}\left(B, \mu_{B, h}\right)(1 \leq p<\infty)$ if, for any increasing sequence of finite dimensional subspaces $\left(E_{n}\right)$ of $H$ having a dense union in $H$, the restriction $\left.F\right|_{E_{n}}$ is in $L^{p}\left(E_{n}, \mu_{E_{n}, h}\right)$, and if the sequence of functions $i_{E_{n}}\left(\left.F\right|_{E_{n}}\right)$ (which are elements of $L^{p}\left(B, \mu_{B, h}\right)$ defined in Theorem 1.1), is a Cauchy sequence in $L^{p}\left(B, \mu_{B, h}\right)$. The limit, which is independent of the sequence $\left(E_{n}\right)$ is called the stochastic extension of $F$ and is here often denoted by $\widetilde{F}$.

The stochastic extension notion is introduced by L. Gross in [19], but involving a convergence in probability instead of a $L^{p}\left(B, \mu_{B, h}\right)$ convergence. The terminology stochastic extension is taken from [19]. Also note that an equivalent form of Definition 1.6 above is given in [3] (Definition 4.4). The simplest example is the case where the symbol $F$ is uniformly continuous for the norm of $B$ restricted to $H$ (see Section 8 in [3]). We shall use also Theorem 8.8 in [3], recalled in Theorem 2.9 in the next section. Also remind that any polynomial function on $H$, that is, any polynomial expression of $f \rightarrow\langle f, u\rangle$ with $u \in H$, has a stochastic extension which is the corresponding Wick polynomial.

We can now define anti-Wick operators (see also [10, 2]) using the Segal Bargmann transform $T_{h}^{F W} f$ defined below Definition 2.2. for any element $f \in \mathcal{F}_{s}\left(H_{\mathbf{C}}\right)$.

Definition 1.7. Let $H$ be a separable real Hilbert space of infinite dimension and $h>0$. Set $B$ a Wiener extension of $H$ and set $\mu_{B, h}$ the measure defined in Section 2.1, and $\mu_{B^{2}, h}$ the similar measure on $H^{2}$. Let $F$ be a bounded continuous function on $H^{2}$, admitting a stochastic extension $\widetilde{F}$ in $L^{2}\left(B^{2}, \mu_{B^{2}, h}\right)$. Then, the operator $O p_{h}^{A W}(F)$ is the unique operator such that, for each $f$ and $g$ in $\mathcal{F}_{s}\left(H_{\mathbf{C}}\right)$ :

$$
<O p_{h}^{A W}(F) f, g>=\int_{B^{2}} \widetilde{F}(x) T_{h}^{F W} f(x) \overline{T_{h}^{F W} g(x)} d \mu_{B^{2}, h}(x) .
$$

This operator is bounded and satisfies:

$$
\left\|O p_{h}^{A W}(F)\right\| \leq\|\widetilde{F}\|_{\infty} \leq\|F\|_{\infty} .
$$

Let us mention that this definition can be extended to the case of unbounded symbols. In that case, the corresponding anti-Wick operator is unbounded and its domain needs therefore to be specified. This 
is precisely the case of the example (Example 1) below. This is also the case when the symbol $F$ is a polynomial function and $\widetilde{F}$ is the corresponding Wick polynomial.

Example 1. In some spin boson models, an important role is played by some Hamiltonian acting in the Hilbert space $\mathcal{F}_{s}\left(H_{\mathbf{C}}\right) \otimes \mathcal{H}_{s p}$ where $H$ is an infinite dimensional real Hilbert space (the configuration space for the single particle states) and $\mathcal{H}_{s p}$ is finite dimensional. Some Hamiltonians of this type are defined for example in $[53,29,16]$.

One shows (see [16] or [53]) that this Hamiltonian has an eigenfunction $U$ with unit norm, with an eigenvalue located at the bottom of the spectrum of the Hamiltonian. This fact comes from [27] or [53]. If some coupling constant appearing in the operator is small enough then the ground state is unique up to a constant multiplicative factor. One also shows in [29] that, for each $R>1, U$ is in $\mathcal{F}_{s}^{R}\left(H_{\mathbf{C}}\right) \otimes \mathcal{H}_{s p}$.

One now chooses a Hermitian basis $S^{(\lambda)}(1 \leq \lambda \leq d)$ of $\mathcal{H}_{s p}$. We denote by $U_{\lambda}$ the components of the ground state $U$ in this basis. Namely,

$$
U=\sum_{\lambda=1}^{d} U_{\lambda} \otimes S^{(\lambda)}
$$

Scattering theory involves sometimes an operator called scattering identification. This identification operator is defined for the case of spin boson models in $[29,54,13,16]$.

The scattering identification $J$ is defined for all $\varphi$ in $\mathcal{F}_{s}^{R}\left(H_{\mathbf{C}}\right)$ (with $R>1$ ) by:

$$
J \varphi=\sum_{\lambda=1}^{d} I\left(U_{\lambda}, \varphi\right) \otimes S^{(\lambda)} .
$$

By Proposition 1.4, $J$ is bounded from $\mathcal{F}_{s}^{R}\left(H_{\mathbf{C}}\right)$ into $\mathcal{F}_{s}\left(H_{\mathbf{C}}\right) \otimes \mathcal{H}_{s p}$ for all $R>1$.

We know that $T_{h}^{F H} U_{\lambda}$ is a regular function on $H^{2}$. It has a stochastic extension which is $T_{h}^{F W} U_{\lambda}$ (Proposition 2.8). However, $T_{h}^{F H} U_{\lambda}$ is not bounded. Nevertheless the integral (1.11) makes sense with the symbol $T_{h}^{F H} U_{\lambda}$ if $f$ is in $\mathcal{F}_{s}^{R}\left(H_{\mathbf{C}}\right)$ for some $R>1$ since the pointwise product $T_{h}^{F W} U_{\lambda}(\cdot) T_{h}^{F W} f(\cdot)$ is the image by $T_{h}^{F W}$ of $I\left(U_{\lambda}, f\right)$ (Theorem 1.5) and $I\left(U_{\lambda}, f\right)$ is a well defined element of $\mathcal{F}_{s}\left(H_{\mathbf{C}}\right)$ by Proposition 1.4. Thus, the anti-Wick operator associated with the symbol $T_{h}^{F H} U_{\lambda}$ makes sense. It is an unbounded operator and its domain contains the union of the $\mathcal{F}_{s}^{R}\left(H_{\mathbf{C}}\right)$.

Theorem 1.8. Let $R>1$. With the above notations, one has:

$$
I\left(U_{\lambda}, \varphi\right)=O p_{h}^{A W}\left(T_{h}^{F H} U_{\lambda}\right) \varphi
$$

for any $\varphi$ in $\mathcal{F}_{s}^{R}\left(H_{\mathbf{C}}\right)$.

Proof of Theorem 1.8. According to Proposition 2.3, we have for all $\varphi$ in $\mathcal{F}_{s}^{R}\left(H_{\mathbf{C}}\right)$ with $R>1$ and for all $g$ in $\mathcal{F}_{s}\left(H_{\mathbf{C}}\right)$ :

$$
<I\left(U_{\lambda}, \varphi\right), g>=\int_{B^{2}}\left(T_{h}^{F W} I\left(U_{\lambda}, \varphi\right)\right)(x) \overline{\left(T_{h}^{F W} g\right)(x)} d \mu_{B^{2}, h}(x) .
$$

In view of Theorem 1.5, one sees:

$$
<I\left(U_{\lambda}, \varphi\right), g>=\int_{B^{2}}\left(T_{h}^{F W} U_{\lambda}\right)(x)\left(T_{h}^{F W} \varphi\right)(x) \overline{\left(T_{h}^{F W} g\right)(x)} d \mu_{B^{2}, h}(x) .
$$


Note that the above integral makes sense for the foregoing reasons. Equality (1.14) then follows from the definition of $O p_{h}^{A W}$ in (1.11).

In that respect, the scattering identification operator for spin boson type models is essentially, under the Segal Bargmann transform, a pointwise multiplication. In direct relation with this fact, Theorem 1.8 says that the scattering identification is an anti-Wick operator with a symbol equal to a Segal-Bargmann transform of the ground state.

Wick quantization. Regarding now the Wick quantization, it goes in the opposite direction when there are no supplementary hypotheses. That is to say, given any bounded operator $A$ in $\mathcal{F}_{s}\left(H_{\mathbf{C}}\right)$, the Wick quantization defines a (symbol) function $\sigma_{h}^{\text {wick }}(A)$ on the phase space $H^{2}$. The definition of this symbol is requiring the use of coherent states.

Coherent states $\Psi_{X h}$ are elements of the Fock space $\mathcal{F}_{s}\left(H_{\mathbf{C}}\right)$ indexed by $X=(q, p) \in H^{2}$ and depending on $h>0$, defined as:

$$
\Psi_{X, h}=e^{-\frac{i}{\sqrt{h}} \Phi_{S}(J X)} \Psi_{0}
$$

where $J(a, b)=(-b, a)$ for $(a, b) \in H^{2}$ and $\Phi_{S}(\cdot)$ is the Segal field (see [46]).

Then we call Wick symbol of an operator $A$ in $\mathcal{F}_{s}\left(H_{\mathbf{C}}\right)$ with a domain containing the coherent states $\Psi_{X, h}\left(X \in H^{2}\right.$ and $\left.h>0\right)$, the following function defined on $H^{2}$ by:

$$
\sigma_{h}^{\text {wick }}(A)(X)=<A \Psi_{X, h}, \Psi_{X, h}>, \quad X \in H^{2},
$$

(the scalar product is linear with respect to the first variable).

The mapping $A \rightarrow \sigma_{h}^{\text {wick }}(A)$ is not onto the set of all bounded continuous functions. It is however onto the set of functions defined below (Definition 1.9) and introduced in [31].

Definition 1.9. For each real separable Hilbert space $H$ and for each nonnegative quadratic form $Q$ on $H^{2}$, let $S\left(H^{2}, Q\right)$ be the class of functions $f \in C^{\infty}\left(H^{2}\right)$ such that there exists $C(f)>0$ satisfying, for any integer $m \geq 0$,

$$
\left|\left(d^{m} f\right)(x)\left(U_{1}, \ldots, U_{m}\right)\right| \leq C(f) Q\left(U_{1}\right)^{1 / 2} \ldots Q\left(U_{m}\right)^{1 / 2}
$$

The smallest constant satisfying (1.17) is denoted by $\|f\|_{Q}$.

In the finite dimensional case, the idea of defining a class of symbols for quantization purposes, in this way, that is to say with the help of a quadratic form on the phase space, goes back to Hörmander [28] and Unterberger [55]. It is proved in [31] that any function belonging to the above space has a stochastic extension.

The following result is proved in [4] (Theorem 2.2).

Proposition 1.10. Let $F \in S\left(H^{2}, Q\right)$ (the class of symbols of Definition 1.9) where $Q$ is a non negative quadratic form on $H^{2}$ and $Q(X)=<A_{Q} X, X>$ for all $X \in H^{2}$ with $A_{Q}$ being trace class. Fix $h>0$. Then, there exists a bounded operator $B_{h}$ such that the Wick symbol of $B_{h}$ is $F$. Thus, we write $B_{h}=$ $O p_{h}^{\text {wick }}(F)$. In addition, the following estimates holds true:

$$
\left\|O p_{h}^{w i c k}(F)\right\| \leq e^{\frac{h}{2} \operatorname{Tr} A_{Q}}\|F\|_{Q} .
$$


Point ii) of Theorem 2.2 in [4] also shows that for any polynomial $F$ on $H^{2}$, there exists an operator $B_{h}$ (generally) unbounded, but with a domain containing coherent states whose Wick symbol is the polynomial $F$. In this particular standard case, one usually refers to Wick (normal) ordering rather than Wick quantization. It is however meaningless for a continuous function (for example a function in $\left.S\left(H^{2}, Q\right)\right)$ to refer to the Wick normal ordering.

Example 2. The time dynamics evolution of the (spin photon) NMR model is studied with Proposition 1.10. Recall that the model is defined with a Hamiltonian $H(h)$ with a parameter $h>0$ acting, as for other spin boson type models, in a Hilbert space of the form $\mathcal{F}_{s}\left(H_{\mathbf{C}}\right) \otimes \mathcal{H}_{s p}$. Here, in the QED framework, $H$ is the space of all $f$ in $L^{2}\left(\mathbb{R}^{3}, \mathbb{R}^{3}\right)$ satisfying $k \cdot f(k)=0$ almost everywhere for $k \in \mathbb{R}^{3}$ and, if there are $P$ particules of spin $1 / 2$ then $\mathcal{H}_{s p}=\left(\mathbb{C}^{2}\right)^{\otimes P}$. See [6] or [47] in a more physical viewpoint.

A spin observable is an operator $I \otimes S$ where $S \in \mathcal{L}\left(\mathcal{H}_{s p}\right)$. The Wick symbol notion for operators in $\mathcal{F}_{s}\left(H_{\mathbf{C}}\right)$ is then easily extended for operators in $\mathcal{F}_{s}\left(H_{\mathbf{C}}\right) \otimes \mathcal{H}_{s p}$. In particular, if $A$ is an operator in $\mathcal{F}_{s}\left(H_{\mathbf{C}}\right) \otimes \mathcal{H}_{s p}$ then its Wick symbol $\sigma_{h}^{\text {wick }}(A)$ is a function defined on $H^{2}$ taking values in $\mathcal{L}\left(\mathcal{H}_{s p}\right)$.

Then, with these notations, the function defined on $H^{2}$ :

$$
A(\cdot, t, h)=\sigma_{h}^{w i c k}\left(e^{i \frac{t}{h} H(h)}(I \otimes S) e^{-i \frac{t}{h} H(h)}\right)
$$

is studied in [4] (Theorem 1.3) for any $S \in \mathcal{L}\left(\mathcal{H}_{s p}\right)$.

In [4] (formula (1.20)), a non negative quadratic form $Q_{t}$ is introduced on the space $H^{2}$ for all $t>0$, such that the associated operator $A_{Q_{t}}$ is trace class. It is proved in [4] (Theorem 1.3) that the function $X \rightarrow A(t, X, h)$ belongs to $S\left(H^{2}, 4 Q_{t}\right)$ with values in $\mathcal{L}\left(\mathcal{H}_{s p}\right)$. Its norm is bounded when $t$ varies in a compact set. An asymptotic expansion in power of the parameter $h$ for this function is additionally given as $h \rightarrow 0$, in [4].

Considering only the first term of the expansion, one then recovers the Bloch equations [11], which play the role of the classical mechanics equations for the RMN issues. Moreover, in virtue of Theorem 2.4 in [4], one obtains that $e^{i \frac{t}{h} H(h)}(I \otimes S) e^{-i \frac{t}{h} H(h)}$ is an anti-Wick operator associated with a symbol $F_{t}$ which belongs to $S\left(H^{2}, 4 Q_{t}\right)$ for all time $t>0$.

Example 3. In [5], one considers the operator $e^{-i \frac{t}{h} H(h)}$ for the same model, the NMR model. It is proved ([5], Theorem 7.1) that:

$$
e^{-i \frac{t}{h} H(h)}=\left[e^{-i \frac{t}{h} H_{p h}} \otimes I\right] O p_{h}^{A W}(F(t, \cdot, h)),
$$

where the symbol $F(t, \cdot, h)$ belongs to the class $S\left(H^{2}, Q_{t}, \mathcal{L}\left(\mathcal{H}_{s p}\right)\right)$ for all time $t>0$. Here, the unitary group $e^{-i \frac{t}{h} H_{p h}}$ is the photon time free evolution operator which is also a Bogoliubov operator.

Consequently, this justifies our interest for bounded operators and especially bounded operators with a symbol which belongs to the class $S\left(H^{2}, Q\right)$.

Weyl quantization. The third quantization considered in this paper is the Weyl one. It is commonly used in finite dimension $([28])$ and extended in the Fock space setting (see $[3,4,5]$ and see also Krée-Rạczka [33]). The simplest way to define Weyl quantization in infinite dimension is to first define the quantized operator as a quadratic form (Definition 2.4 in [5]). Extensions and norm estimates issues are considered in $[3,4,5]$. We proved the existence of a bounded extension only when the symbol $F$ lies in the class 
$S\left(H^{2}, Q\right)$ of Definition 1.9 if the quadratic form $Q$ is non negative on $H^{2}$ and where $Q(X)=<A_{Q} X, X>$, $X \in H^{2}$, with $A_{Q}$ being trace class. In that case, the extension is the Weyl operator associated with the symbol $F$ and it is denoted by $O p_{h}^{\text {weyl }}(F)$.

Note that the Wigner measures considered in [2] are limits of Wigner functions used for the Weyl calculus definition. These Wigner measures enable in [2] the description of the time dynamics of any arbitrary initial states.

The relations between the three symbols of a same operator are given using the heat operator (see Gross [22] and Hall [23] in infinite dimension). Fix $h>0$. The heat operator is defined for each continuous bounded function $F$ on $H^{2}$ admitting a stochastic extension $\widetilde{F}$ in $L^{1}\left(B^{2}, \mu_{B, h}\right)$ (see Definition 1.6) and for each $h>0$ by:

$$
\left(H_{h} F\right)(X)=\int_{B^{2}} \widetilde{F}(X+Y) d \mu_{B^{2}, h}(Y),
$$

for any $X \in H^{2}$. In particular, one can define $H_{h} F$ if $F \in S\left(H^{2}, Q\right)$, with a non negative trace class operator $A_{Q}$. It is proved in [4] (formula (2.13) that, if $A=O p_{h}^{A W}(F)$ where $F$ on $S\left(H^{2}, Q\right)$ as above, then:

$$
\sigma_{h}^{w i c k}(A)=H_{h} F \text {. }
$$

It is also proved in [5] (formula (33)) that, for each $G \in S\left(H^{2}, Q\right)$ where the quadratic form $Q$ satisfies the same assumptions as before:

$$
\sigma_{h}^{\text {wick }}\left(O p_{h}^{\text {weyl }}(G)\right)=H_{\frac{h}{2}} G .
$$

Therefore, if $A=O p_{h}^{A W}(F)$ with $F \in S\left(H^{2}, Q\right)$ as above, then we have:

$$
A=O p_{h}^{\text {weyl }}\left(H_{\frac{h}{2}} F\right) .
$$

Consequently, any anti-Wick operator with a symbol in $S\left(H^{2}, Q\right)$ is also a Weyl operator with another symbol but still in the same class.

As in finite dimension, one of the major interest of the Weyl calculus in the infinite dimension context is the symplectic group covariance property. First, one defines, as in finite dimension, a unitary operator $U_{\chi}$ in $\mathcal{F}_{s}\left(H_{\mathbb{C}}\right)$ which belongs to the class of operators called Bogoliubov operators (and called metaplectic operators in finite dimension), for any symplectic mapping $\chi$ acting in the phase space $H^{2}$ such that $\chi^{\star} \chi-I$ is Hilbert Schmidt (see Shale [49] or Lascar [38]). Then:

$$
U_{\chi}^{\star} O p_{h}^{\text {weyl }}(F) U_{\chi}=O p_{h}^{\text {weyl }}(F \circ \chi),
$$

for any $F \in S\left(H^{2}, Q\right)$, if $\chi^{\star} \chi-I$ is trace class (see [5], Theorem 4.4). This result is actually proved in [38] in the case of a quadratic Hamiltonian, with different notations.

\subsection{Wick and Weyl symbols for composition of operators.}

We shall give explicit formulas for the Wick symbol (defined in (1.16)) of the composition $A \circ B$ where $A$ and $B$ are two bounded operators in $\mathcal{F}_{s}\left(H_{\mathbf{C}}\right)$, as the sum of an absolutely convergent series. In finite dimension, this is a result of Mizrahi [43] and Appleby [7]. Note that, for this result, namely Theorem 
1.11, the operators $A$ and $B$ are supposed to be any bounded operators and not necessarily to be defined with a quantization.

If $H$ is a separable, real Hilbert space and if $\left(e_{j}\right)$ is a Hilbert basis of $H$, we define, for all multi-index $\alpha=\left(\alpha_{j}\right)$ (which means $\alpha_{j}=0$ except for a finite number of values of $j$ ), two differential operators on $H^{2}$, denoting by $(q, p)$ the variable of $H^{2}$ :

$$
\left(\partial_{q} \pm i \partial_{p}\right)^{\alpha}=\prod_{j}\left(\frac{\partial}{\partial q_{j}} \pm i \frac{\partial}{\partial p_{j}}\right)^{\alpha_{j}}
$$

Theorem 1.11. For every bounded operators $A$ and $B$ in $\mathcal{F}_{s}\left(H_{\mathbf{C}}\right)$, the Wick symbols $F$ and $G$ of $A$ and $B$ are $C^{\infty}$ functions on $H^{2}$. For each $k \geq 0$, the following series, a priori defined using a Hilbertian basis $\left(e_{j}\right)$ of $H$ :

$$
C_{k}^{w i c k}(F, G)=2^{-k} \sum_{|\alpha|=k}\left(\frac{1}{\alpha !}\right)\left(\partial_{q}-i \partial_{p}\right)^{\alpha} F\left(\partial_{q}+i \partial_{p}\right)^{\alpha} G
$$

is absolutely convergent and its sum $C_{k}^{\text {wick }}(F, G)$ is independent of the basis. We have, for all $X$ in $H^{2}$ :

$$
\sigma_{h}^{w i c k}(A \circ B)(X)=\sum_{k=0}^{\infty} h^{k} C_{k}^{w i c k}\left(\sigma_{h}^{w i c k}(A), \sigma_{h}^{w i c k}(B)\right)(X),
$$

where the series is absolutely convergent.

This theorem will be proved in Section 3 .

See [7] or [43] in the case of finite dimension. See also [2], formula (15)(i). If the operators $A$ and $B$ act in $\mathcal{F}_{s}\left(H_{\mathbf{C}}\right) \otimes E$ where $E$ is a Hilbert of finite dimension then the functions $F$ and $G$ are defined on $H^{2}$, taking values in $\mathcal{L}(E)$ and the product in the right hand side of (1.23) is a composition of operators. Note that, if one of the functions $F$ or $G$ is scalar valued, one has

$$
C_{1}^{\text {wick }}(F, G)-C_{1}^{\text {wick }}(G, F)=i^{-1}\{F, G\} .
$$

One denotes by $\sigma$ the symplectic form on $H^{2}$ defined by $\sigma((x, \xi),(y, \eta))=y \cdot \xi-x \cdot \eta$ and by $\{F, G\}$ the Poisson bracket of two functions $F$ and $G$ in $C^{1}\left(H^{2}\right)$, defined by $\{F, G\}(X)=-\sigma(d F(X), d G(X))$.

In Section 4, we study the composition for the Weyl quantization. One shows in [5] that, if $F$ and $G$ are in $S\left(H^{2}, Q\right)$ and if $A_{Q}$ is trace class then the composition $O p_{h}^{\text {weyl }}(F) \circ O p_{h}^{\text {weyl }}(G)$ can be written as $O p_{h}^{\text {weyl }}\left(K_{h}^{\text {weyl }}(F, G)\right)$ with $K_{h}^{\text {weyl }}(F, G)$ belonging to $S\left(H^{2}, 4 Q\right)$. We shall give an expression of $K_{h}^{\text {weyl }}(F, G)$ written as an absolutely convergent series.

One defines a differential operator $\sigma\left(\nabla_{1}, \nabla_{2}\right)$ on $H^{2} \times H^{2}$ by:

$$
\sigma\left(\nabla_{1}, \nabla_{2}\right) F=\sum_{j} \frac{\partial^{2} F}{\partial y_{j} \partial \xi_{j}}-\frac{\partial^{2} F}{\partial x_{j} \partial \eta_{j}}
$$

where $(x, \xi, y, \eta)$ is the variable in $H^{2} \times H^{2}$. This operator is defined only for functions $F$ for which the series converges. For all integers $k$ and all functions $F$ and $G$ such that the series below makes sense, set:

$$
C_{k}^{\text {weyl }}(F, G)(X)=\frac{1}{(2 i)^{k} k !} \sigma\left(\nabla_{1}, \nabla_{2}\right)^{k}(F \otimes G)(X, X) .
$$


Theorem 1.12. Let $Q$ be a nonnegative quadratic form on $H^{2}$ where $A_{Q}$ is trace class. Let $F$ and $G$ be two functions in $S\left(H^{2}, Q\right)$. Then,

i) For each $k \geq 0$, we have:

$$
\left\|C_{k}^{\text {weyl }}(F, G)\right\|_{4 Q} \leq\|F\|_{Q}\|G\|_{Q} \frac{\left(\operatorname{Tr} A_{Q}\right)^{k}}{2^{k} k !}
$$

The series

$$
K_{h}^{w e y l}(F, G)(X)=\sum_{k=0}^{\infty} h^{k} C_{k}^{\text {weyl }}(F, G)
$$

is absolutely convergent and defines a function $K_{h}^{w e y l}(F, G)$ in $S\left(H^{2}, 4 Q\right)$.

ii) For all integers $M$, one can write

$$
K_{h}^{w e y l}(F, G)=\sum_{k=0}^{M} h^{k} C_{k}^{w e y l}(F, G)+h^{M+1} R_{M}^{\text {weyl }}(F, G, h)
$$

with:

$$
\left\|R_{M}^{w e y l}(F, G, h)\right\|_{4 Q} \leq\|F\|_{Q}\|G\|_{Q} \frac{\left(\operatorname{Tr} A_{Q}\right)^{M+1}}{(M+1) !} e^{(h / 2) \operatorname{Tr} A_{Q}} .
$$

iii) One has:

$$
O p_{h}^{w e y l}(F) \circ O p_{h}^{\text {weyl }}(G)=O p_{h}^{\text {weyl }}\left(K_{h}^{w e y l}(F, G)\right) .
$$

This theorem will be proved in Section 4.

\section{Segal Bargmann transformation and Wick product.}

\subsection{Segal isomorphism.}

We recall, for any Hilbert space $H$ with Wiener extension $B$, how the Fock space $\mathcal{F}_{s}\left(H_{\mathbf{C}}\right)$ is made isomorphic to $L^{2}\left(B, \mu_{B, h}\right)$. This will fix some notations used in the sequel.

First, one chooses a Hilbertian basis $\left(e_{j}\right)$ of $H$. For all multi-indices $\alpha$ (a map from $\mathbb{N}$ into $\mathbb{N}$ with $\alpha_{j}=0$ except for a finite number of indices), one defines an element $u_{\alpha}$ of $\mathcal{F}_{s}\left(H_{\mathbf{C}}\right)$ by:

$$
u_{\alpha}=(\alpha !)^{-\frac{1}{2}}\left(\prod_{j}\left(a^{\star}\left(e_{j}\right)\right)^{\alpha_{j}}\right) \Psi_{0}=(\alpha !)^{-\frac{1}{2}}\left(a^{\star}(e)\right)^{\alpha} \Psi_{0}
$$

where $\Psi_{0}$ is the vacuum state. We know that $\left(u_{\alpha}\right)$ constitutes a Hilbertian basis of $\mathcal{F}_{s}\left(H_{\mathbf{C}}\right)$. We also have:

$$
u_{\alpha}=(\alpha !)^{-\frac{1}{2}}(|\alpha| !)^{\frac{1}{2}} \Sigma\left(\otimes_{j} e_{j}^{\alpha_{j}}\right)
$$


where $\Sigma$ stands for the orthogonal projection from the Fock space $\mathcal{F}\left(H_{\mathbf{C}}\right)$ to its symmetrization $\mathcal{F}_{s}\left(H_{\mathbf{C}}\right)$. We naturally set $u_{0}=\Psi_{0}$, the vacuum state.

Under $S_{h}$, the image of $f \in H^{(0)}$ (see 1.1) is a constant function (belonging to $L^{2}\left(B, \mu_{B, h}\right)$ ) equaling the constant number $C$ if $f=C \Psi_{0}$ with $\Psi_{0}$.

The image of the isomorphism $S_{h}$ restricted to $H^{(1)}$ is isomorphic to $H_{\mathbf{C}}$ and is sometimes called Gaussian random process indexed by $H_{\mathbf{C}}$ (see for example $[50,32,44]$ ). We remind some details concerning this notion. We recall ([36]) that, for all $a$ in $B^{\prime} \subset H$, the function $B \ni x \mapsto a(x)$ belongs to $L^{2}\left(B, \mu_{B, h}\right)$, with a norm equal to $h^{\frac{1}{2}}|a|$. Thus, the map associating with every $a \in B^{\prime}$ the above function $B \ni x \mapsto a(x)$ considered as an element of $L^{2}\left(B, \mu_{B, h}\right)$, can be extended by density to a mapping $a \mapsto \ell_{a}$ from $H$ into $L^{2}\left(B, \mu_{B, h}\right)$.

Polynomial expressions with respect to the $\ell_{e_{j}}$ (view as random variables) are called Wick polynomials. Still in the probabilistic setting, the Wick polynomial function is also given with a Wiener multiple integral, see [30, 41, 44, 37].

Definition 2.1. One denotes by $S_{h}$ the continuous linear mapping from $\mathcal{F}_{s}^{\text {fin }}\left(H_{\mathbf{C}}\right)$ into $L^{2}\left(B, \mu_{B, h}\right)$ satisfying for every multi-indices $\alpha$ :

$$
S_{h} u_{\alpha}=\prod_{\alpha_{j} \neq 0} H_{\alpha_{j}}\left(\frac{\ell_{e_{j}}(x)}{\sqrt{h}}\right)
$$

where the $H_{n}$ are the Hermite polynomials on $\mathbb{R}$ (chosen as an orthonormal system in $L^{2}\left(\mathbb{R}, \mu_{\mathbb{R}, 1}\right.$ ).

One shows (see [32]) that $S_{h}$ is extended to an unitary isomorphism (Segal isomorphism) from $\mathcal{F}_{s}\left(H_{\mathbf{C}}\right)$ into $L^{2}\left(B, \mu_{B, h}\right)$ and that

$$
L^{2}\left(B, \mu_{B, h}\right)=\underset{m \geq 0}{\oplus} S_{h} H^{(m)} .
$$

This equality is usually called Wiener-Itô chaos decomposition ([56, 30, 25, 32, 41, 44, 50]).

The space $S_{h} H^{(m)}$ of Wiener chaos of order $m$ can also be given with Itô multiple integrals of order $m$ $([41,37,25])$.

\subsection{Segal Bargmann transform.}

Let $H$ be a separable real Hilbert space of infinite dimension and let $\mathcal{F}_{s}\left(H_{\mathbf{C}}\right)$ be the symmetrized Fock space over $H_{\mathbf{C}}$ the complexification of $H$. We define two Segal Bargmann transforms of any arbitrary $f \in \mathcal{F}_{s}\left(H_{\mathbf{C}}\right)$. The first one is an element of $\mathcal{F}_{s}\left(H_{\mathbf{C}} \times H_{\mathbf{C}}\right)$ and is denoted by $T^{F F} f$. The second one is a function on $H^{2}$ and is denoted by $T_{h}^{F H} f$.

Coherent states (1.15) are involved in order to define $T_{h}^{F H} f$. We denote by $\Gamma(\cdot)$ the functor mapping any operator $U$ from $H_{\mathbf{C}}$ into $H_{\mathbf{C}} \times H_{\mathbf{C}}$ to an operator $\Gamma(U)$ from $\mathcal{F}_{s}\left(H_{\mathbf{C}}\right)$ into $\mathcal{F}_{s}\left(H_{\mathbf{C}} \times H_{\mathbf{C}}\right)$ (see [46]).

Definition 2.2. Let $H$ be a real separable space of infinite dimension.

i) The mapping $T^{F F}$ acting from $\mathcal{F}_{s}\left(H_{\mathbf{C}}\right)$ into $\mathcal{F}_{s}\left(H_{\mathbf{C}} \times H_{\mathbf{C}}\right)$ is defined by:

$$
T^{F F}=\Gamma\left(U_{-}\right), \quad U_{ \pm}(X)=(1 / \sqrt{2})(X, \pm i X), X \in H_{\mathbf{C}} .
$$


ii) For any fixed $h>0, T_{h}^{F H}(h>0)$ maps any element $f$ in $\mathcal{F}_{s}\left(H_{\mathbf{C}}\right)$ to a function $T_{h}^{F H} f$ defined on $H^{2}$ by:

$$
\left(T_{h}^{F H} f\right)(X)=\frac{<f, \Psi_{X, h}>}{<\Psi_{0}, \Psi_{X, h}>}=e^{\frac{|X|^{2}}{4 h}}<f, \Psi_{X, h}>
$$

The second equality in (2.4) comes from the following identity: for all $X$ and $Y$ in $H^{2}$,

$$
<\Psi_{X h}, \Psi_{Y h}>=e^{-\frac{1}{4 h}\left(|X-Y|^{2}\right)+\frac{i}{2 h} \sigma(X, Y)},
$$

where $\sigma$ is the symplectic form $\sigma\left((q, p),\left(q^{\prime}, p^{\prime}\right)\right)=p \cdot q^{\prime}-q \cdot p^{\prime}$.

A third Segal Bargmann transform $T_{h}^{F W}$ can be defined using a Wiener extension space $B$ of the Hilbert space $H\left(B^{2}\right.$ is a Wiener extension of $\left.H^{2}\right)$.

For the sake of clarity, we distinguish in notations for the notions concerning the Fock space case $\mathcal{F}_{s}\left(H_{\mathbf{C}}\right)$ and those concerning the Fock space case $\mathcal{F}_{s}\left(H_{\mathbf{C}} \times H_{\mathbf{C}}\right)$, by using an additional index or exponent 2 in the second case. For instance, in $\mathcal{F}_{s}\left(H_{\mathbf{C}} \times H_{\mathbf{C}}\right)$, the scalar product is denoted by $\langle\cdot, \cdot\rangle_{2}$ (recall that it is linear with respect to the left variable), the functor $\Gamma$ is written as $\Gamma_{2}$ and $S_{h, 2}$ stands for the Segal isomorphism.

With theses notations, we set:

$$
T_{h}^{F W}=S_{h, 2} \circ T_{h}^{F F}
$$

where the Segal isomorphism $S_{h, 2}: \mathcal{F}_{s}\left(H_{\mathbf{C}} \times H_{\mathbf{C}}\right) \rightarrow L^{2}\left(B^{2}, \mu_{B^{2}, h}\right)$ (see Definition 2.1). Thus, $T_{h}^{F W} f$ is an element of $L^{2}\left(B^{2}, \mu_{B^{2}, h}\right)$. The image under $S_{h, 2}$ can be named the complex wave representation whereas Fock symmetrized space $\mathcal{F}_{s}\left(H_{\mathbf{C}}\right)$ and $L^{2}\left(B, \mu_{B, h}\right)$ can respectively be called particle representation and real wave representation (see [8]).

We shall see the connection between the two Segal Bargmann $T_{h}^{F W} f$ and $T_{h}^{F H} f$ in Subsection 2.4.

The following proposition is a consequence of Definition 2.2 together with the fact that $S_{h, 2}$ is a unitary isomorphism.

Proposition 2.3. i) The operator $T^{F F}$ is a partial isometry from $\mathcal{F}_{s}\left(H_{\mathbf{C}}\right)$ into $\mathcal{F}_{s}\left(H_{\mathbf{C}} \times H_{\mathbf{C}}\right)$. For all $R>1$ and for any $f$ in $\mathcal{F}_{s}^{R}\left(H_{\mathbf{C}}\right)$, we have with the notation (1.5):

$$
\left\|T^{F F} f\right\|_{R, 2}=\|f\|_{R} .
$$

ii) The map $T_{h}^{F W}$ is a partial isometry from $\mathcal{F}_{s}\left(H_{\mathbf{C}}\right)$ into $L^{2}\left(B^{2}, \mu_{B^{2}, h}\right)$.

The next Proposition is standard but it is recalled here since it is used in Subsection 2.3.

Proposition 2.4. For any $V$ in $H$ being identified with the element $(V, 0)$ and for all $X=(q, p)$ in $H^{2}$, we have:

$$
\begin{aligned}
T^{F H}\left(a^{\star}(V) f\right)(q, p) & =\frac{1}{\sqrt{2 h}}(V \cdot q-i V \cdot p)\left(T^{F H} f\right)(q, p), \\
T^{F H}(a(V) f)(q, p) & =\sqrt{\frac{h}{2}}\left(V \cdot\left(\partial_{q}+i \partial_{p}\right)\right)\left(T^{F H} f\right)(q, p) .
\end{aligned}
$$


Proof of Proposition 2.4. Set $(q+i p)^{\otimes m}=(q+i p) \otimes \cdots \otimes(q+i p)$ ( $m$ times). Using the formulas (X.63) of [46], we have:

$$
\begin{aligned}
a(V)(q+i p)^{\otimes^{m}} & =\sqrt{m}(V \cdot(q+i p))(q+i p))^{\otimes^{m-1}}, \\
a^{\star}(V)(q+i p)^{\otimes^{m}} & =\frac{1}{\sqrt{m+1}} \sum_{k=0}^{m}(q+i p)^{\otimes k} \otimes V \otimes(q+i p)^{\otimes^{m-k}} .
\end{aligned}
$$

In other words:

$$
V \cdot\left(\partial_{q}-i \partial_{p}\right)(q+i p)^{\otimes m}=2 \sqrt{m} a^{\star}(V)(q+i p)^{\otimes^{m-1}} .
$$

By (1.15), it follows that:

$$
a(V) \Psi_{q, p, h}=\frac{1}{\sqrt{2 h}} V \cdot(q+i p) \Psi_{q, p, h}
$$

and also that:

$$
a^{\star}(V) \Psi_{q, p, h}=\sqrt{\frac{h}{2}} e^{-\left(|q|^{2}+|p|^{2}\right) / 4 h} V \cdot\left(\partial_{q}-i \partial_{p}\right)\left(e^{\left(|q|^{2}+|p|^{2}\right) / 4 h} \Psi_{q, p, h}\right) .
$$

Equalities (2.7) and (2.8) then follow easily by (2.4).

\subsection{Power series expansions.}

For any $f$ in $\mathcal{F}_{s}\left(H_{\mathbf{C}}\right)$, we give in this Subsection power series expansions of the two functions $T_{h}^{F H} f$ and $T_{h}^{F W} f$ with two different senses of convergence.

Fix a Hilbertian basis $\left(e_{j}\right)$ of $H$. Then define the basis $\left(u_{\alpha}\right)$ of $\mathcal{F}_{s}\left(H_{\mathbf{C}}\right)$ as in (2.1). For each multi-index $\alpha$, also define a function $\Phi_{\alpha, h}$ on $H^{2}$ by:

$$
\Phi_{\alpha, h}(q, p)=(2 h)^{-|\alpha| / 2}(\alpha !)^{-1 / 2} \prod_{j}\left(e_{j} \cdot(q-i p)\right)^{\alpha_{j}} .
$$

The convergence concerning the expansion of the function $T_{h}^{F H} f$ is pointwise and not absolute due to the infinite dimensional setting. Let $\mathcal{M}$ be the set of all multi-indices $\alpha$ and let $\left(I_{N}\right)$ be an increasing sequence of finite subsets of $\mathcal{M}$ with union $\mathcal{M}$.

Proposition 2.5. i) For any $f$ in $\mathcal{F}_{s}\left(H_{\mathbf{C}}\right)$ and for all $X$ in $H^{2}$, we have:

$$
\left(T_{h}^{F H} f\right)(X)=\lim _{N \rightarrow \infty} \sum_{\alpha \in I_{N}}<f, u_{\alpha}>\Phi_{\alpha, h}(X) .
$$

ii) The function $T_{h}^{F H} f$ is Gateaux anti-holomorphic if $H^{2}$ is identified with $H_{\mathbf{C}}$ (when identifying $X=$ $(q, p)$ with $q+i p)$. That is, the restriction of $T_{h}^{F H} f$ to $E$ is anti-holomorphic for any finite dimensional complex subspace $E$ of $H_{\mathbf{C}}$. 
iii) For any square-summable sequence $\left(a_{\alpha}\right)$, there exists a function $F$ on $H^{2}$ satisfying for all $X$ in $H^{2}$ :

$$
F(X)=\lim _{N \rightarrow \infty} \sum_{\alpha \in I_{N}} a_{\alpha} \Phi_{\alpha, h}(X) .
$$

Moreover, there exists $f$ in $\mathcal{F}_{s}\left(H_{\mathbf{C}}\right)$ satisfying $T_{h}^{F H} f=F$.

Proof of Proposition 2.5. Let us first check that:

$$
T_{h}^{F H} u_{\alpha}(X)=e^{\frac{|X|^{2}}{4 h}}<u_{\alpha}, \Psi_{X, h}>=\Phi_{\alpha, h}(X), \quad X=(q, p) \in H^{2} .
$$

This fact is proved by induction on $|\alpha|$. For $\alpha=0$, namely for $u_{0}=\Psi_{0}$ and $\Phi_{0, h}=1$, this is a consequence of (2.4). Then (2.12) is verified by iteration in view of (2.7).

Concerning point i), set for any integers $N$ :

$$
f_{N}=\sum_{\alpha \in I_{N}}<f, u_{\alpha}>u_{\alpha}
$$

According to (2.12), one has for any $X$ in $H^{2}$ :

$$
\sum_{\alpha \in I_{N}}<f, u_{\alpha}>\Phi_{\alpha, h}(X)=e^{\frac{|X|^{2}}{4 h}}<f_{N}, \Psi_{X, h}>
$$

Since $f_{N}$ converges to $f$ in $\mathcal{F}_{s}\left(H_{\mathbf{C}}\right)$ then point i) follows, for any fixed $X$.

ii) Let $E$ be a complex subspace of $H_{\mathbf{C}}$ of finite dimension $m$. Set $\left(w_{1}, \ldots, w_{m}\right)$ a complex orthonormal basis of $E$. Thus, any element $Z$ of $E$ is written as $Z=z_{1} w_{1}+\cdots+z_{m} w_{m}$, with $z_{j}$ in $\mathbf{C}$. Thanks to (2.9) and (2.10), we can then write, for all $Z \in E$ :

$$
T_{h}^{F H} f(Z)=\sum c_{\gamma} \bar{z}^{\gamma}
$$

where the series in the right hand side is converging for all $z$ in $\mathbf{C}^{m}$. The anti-holomorphy property of the restriction of $T_{h}^{F H} f$ to $E$ then follows.

iii) If the sequence $\left(a_{\alpha}\right)$ is square-summable, let $f_{N}$ be the element of $\mathcal{F}_{s}\left(H_{\mathbf{C}}\right)$ defined by:

$$
f_{N}=\sum_{\alpha \in I_{N}} a_{\alpha} u_{\alpha} .
$$

The sequence $\left(f_{N}\right)$ tends to an element $f$ of $\mathcal{F}_{s}\left(H_{\mathbf{C}}\right)$ and the above reasoning shows that:

$$
\lim _{N \rightarrow \infty} \sum_{\alpha \in I_{N}} a_{\alpha} \Phi_{\alpha, h}(X)=T_{h}^{F H} f(X) .
$$

Let us now consider the series expansion of $T_{h}^{F W} f$. One defines a function $\widetilde{\Phi}_{\alpha}$ almost everywhere on $B^{2}$ by:

$$
\widetilde{\Phi}_{\alpha, h}(q, p)=(2 h)^{-|\alpha| / 2}(\alpha !)^{-1 / 2} \prod\left(\ell_{e_{j}}(q)-i \ell_{e_{j}}(p)\right)^{\alpha_{j}} .
$$

The functions $\widetilde{\Phi}_{\alpha, h}$ constitute an orthonormal basis in $L^{2}\left(B^{2}, \mu_{B^{2}, h}\right)$. 
Proposition 2.6. One has:

$$
T_{h}^{F W} u_{\alpha}=\widetilde{\Phi}_{\alpha, h}
$$

Proof. If $\left(e_{j}\right)$ is a Hilbertian basis of $H$ then we use the Hilbertian basis of $H^{2}$ constituted of the $\left(e_{j}, 0\right)$ and $\left(0, e_{j}\right)$. The elements of $\mathcal{F}_{s}\left(H_{\mathbf{C}} \times H_{\mathbf{C}}\right)$ constructed with this basis defined as in $(2.1)$ are denoted by $u_{\alpha \beta}$. We observe that:

$$
\Gamma\left(U_{-}\right) u_{\alpha}=2^{-|\alpha| / 2} \sum_{\beta+\gamma=\alpha} \sqrt{\frac{\alpha !}{\beta ! \gamma !}}(-i)^{|\gamma|} u_{\beta, \gamma} .
$$

According to the Segal isomorphism definition in $(2.2)$, considering $(q, p)$ as the variable of $B^{2}$ and setting $\ell_{\left(e_{j}, 0\right)}(q, p)=\ell_{e_{j}}(q)$ and $\ell_{\left(0, e_{j}\right)}(q, p)=\ell_{e_{j}}(p)$, one checks:

$$
S_{h, 2}\left(u_{\beta, \gamma}\right)(q, p)=\prod_{j, \beta_{j}+\gamma_{j} \neq 0} H_{\beta_{j}}\left(\ell_{e_{j}}(q) / \sqrt{h}\right) H_{\gamma_{j}}\left(\ell_{e_{j}}(p) / \sqrt{h}\right)
$$

where the $H_{k}$ are the Hermite polynomials chosen being an orthonormal basis in $L^{2}\left(\mathbb{R}, \mu_{\mathbb{R}, 1}\right)$. Then we use the following identity, probably standard, for all $(x, y)$ in $\mathbb{R}^{2}$ :

$$
\frac{(x-i y)^{m}}{\sqrt{m !}}=\sum_{p+q=m} \sqrt{\frac{m !}{p ! q !}}(-i)^{q} H_{p}(x) H_{q}(y) .
$$

The proof of the Proposition is then completed.

The result below will be used in Section 3 .

Proposition 2.7. For any $f$ and $g$ in $\mathcal{F}_{s}\left(H_{\mathbf{C}}\right)$, one has in the sense of the $L^{2}\left(B^{2}, \mu_{B^{2}, h}\right)$ Hilbert space convergence:

$$
\begin{gathered}
T_{h}^{F W} f=\lim _{N \rightarrow \infty} \sum_{\alpha \in I_{N}}<f, u_{\alpha}>\widetilde{\Phi}_{\alpha, h} \\
<f, g>=\sum<T_{h}^{F W} f, \widetilde{\Phi}_{\alpha, h}>_{h}<\widetilde{\Phi}_{\alpha, h}, T_{h}^{F W} g>_{h}
\end{gathered}
$$

where the series is absolutely converging.

The above scalar products $\left\langle\cdot, \cdot>\right.$ and $\left\langle\cdot, \cdot>_{h}\right.$ respectively are the canonical $\mathcal{F}_{s}\left(H_{\mathbf{C}}\right)$ and $L^{2}\left(B^{2}, \mu_{B^{2}, h}\right)$ scalar products.

Again, we emphasize that the convergence in $(2.16)$ is the $L^{2}\left(B^{2}, \mu_{B^{2}, h}\right)$ Hilbert space convergence whereas it is a pointwise convergence in $(2.10)$, for all $X$ in $H^{2}$.

Proof of Proposition 2.7. Let $f_{N}$ be the element defined in (2.13). In view of (2.15), one notices that:

$$
T_{h}^{F W} f_{N}=\sum_{\alpha \in I_{N}}<f, u_{\alpha}>\widetilde{\Phi}_{\alpha, h}
$$

According to point $i$ ) of Proposition 2.3, one sees that $T_{h}^{F W} f_{N}$ tends to $T_{h}^{F W} f$ in $L^{2}\left(B^{2}, \mu_{B^{2}, h}\right)$. One then gets equality (2.16). The second equality therefore holds true since the set of functions $\widetilde{\Phi}_{\alpha, h}$ is an orthonormal system in $L^{2}\left(B^{2}, \mu_{B^{2}, h}\right)$. 


\subsection{Connection between $T_{h}^{F H} f$ and $T_{h}^{F W} f$.}

Two Segal Bargmann transforms of $f$, namely $T_{h}^{F H} f$ and $T_{h}^{F W} f$, are previously defined for any $f \in$ $\mathcal{F}_{s}\left(H_{\mathbf{C}}\right)$. One is a function on $H^{2}$ (Gateaux anti-holomorphic on $H^{2}$ ) and the other one is defined almost everywhere on $B^{2}$. One then should specify the relation between these two functions. We underline that one cannot consider the restriction to $H^{2}$ of a measurable function defined on $B^{2}$ since the measure of $H^{2}$ in $B^{2}$ equals to zero.

We prove that $T_{h}^{F W} f$ is a stochastic extension of $T_{h}^{F H} f$ in the sense of Definition 1.6 and that $T_{h}^{F H} f$ is the image of $T_{h}^{F W} f$ using the reproducing kernel defined below.

For all $X=(q, p)$ in $H^{2}$, one defines a function $\ell_{X}$ almost everywhere on $B^{2}$ by $\ell_{X}(Y)=\ell_{q-i p}(y)+$ $i \ell_{q-i p}(\eta)$ where $Y=(y, \eta)$. One also defines almost everywhere a function $Y=(y, \eta) \rightarrow B_{h}(X, Y)$ where:

$$
B_{h}(X, Y)=e^{\frac{1}{2 h} \ell_{X}(Y)}
$$

One knows that the function $Y \rightarrow B_{h}(X, Y)$ belongs to $L^{2}\left(B^{2}, \mu_{B^{2}, h}\right)$ for any $X$ in $H^{2}$ implying that, for all $F$ in $L^{2}\left(B^{2}, \mu_{B^{2}, h}\right)$, the following integral called reproducing kernel:

$$
\left(B_{h} F\right)(X)=\int_{B^{2}} B_{h}(X, Y) F(Y) d \mu_{B^{2}, h}(Y)
$$

defines a function $B_{h} F$ on $H^{2}$.

The link between $T_{h}^{F H} f$ and $T_{h}^{F W} f$ is given by the proposition below.

Proposition 2.8. i) For all $f \in \mathcal{F}_{s}\left(H_{\mathbf{C}}\right)$, the function $T_{h}^{F H} f$ defined on $H^{2}$ admits a stochastic extension in $L^{2}\left(B^{2}, \mu_{B^{2}, h}\right)$. This stochastic extension is $T_{h}^{F W} f$.

ii) One has,

$$
\left(T_{h}^{F H} f\right)(X)=\int_{B^{2}} B_{h}(X, Y) T_{h}^{F W} f(Y) d \mu_{B^{2}, h}(Y)
$$

for every $f$ in $\mathcal{F}_{s}\left(H_{\mathbf{C}}\right)$ and for all $X$ in $H^{2}$.

The proof of point i) relies on the next theorem, which is a variant of Theorem 8.8 in [3] adapted to our current situation.

Theorem 2.9. Let $H$ be an infinite dimensional separable complex Hilbert space and fix $B$ any Wiener extension of $H$. The space $B$ is considered as a real space. Let $F$ be a continuous function on $H$. Assume that:

i) The function $F$ restricted to $E$ is anti-homomorphic where $E$ is any finite dimensional complex subspace of $H$.

ii) For every finite dimensional complex subspace $E$ of $H$, the function $F$ restricted to $E$ belongs to $L^{2}\left(E, \mu_{E, h}\right)$ and its norm is bounded independently of $E\left(\mu_{E, h}\right.$ denotes the Gaussian measure with variance $h$ on $E$ considered as a real space). 
Then, there exists $\widetilde{F}$ a stochastic extension in $L^{2}\left(B, \mu_{B, h}\right)$ of the function $F$ (in the sense of Definition 1.6). Moreover, we have:

$$
\|\widetilde{F}\|_{L^{2}\left(B, \mu_{B, h}\right)} \leq \sup _{E}\left\|R_{E} F\right\|_{L^{2}\left(E, \mu_{E, h}\right)}
$$

where $R_{E} F$ stands for $F$ restricted to $E$ and where the supremum is taken over all the finite dimensional complex subspaces $E$ in $H$.

Proof of Proposition 2.8. i) For all $f$ in $\mathcal{F}_{s}\left(H_{\mathbf{C}}\right)$, we know (Proposition 2.5) that the function $T_{h}^{F H} f$, defined on $H^{2}$ is identified to a function $F$ being Gateaux anti-holomorphic on $H_{\mathbf{C}}$ when $H^{2}$ and $H_{\mathbf{C}}$ are identified. One also knows that this function satisfies hypothesis ii) of Theorem 2.9 and, for all complex subspaces $E$ of $H_{\mathbf{C}}$ :

$$
\left\|R_{E} F\right\|_{L^{2}\left(E, \mu_{E, h}\right)} \leq\|f\| .
$$

This fact comes from the standard properties of the Segal Bargmann transform in finite dimension. According to Theorem 2.9, $T_{h}^{F H} f$ has a stochastic extension $\widetilde{F}$ in $L^{2}\left(B^{2}, \mu_{B^{2}, h}\right)$. Let $\left(f_{N}\right)$ be a sequence in $\mathcal{F}_{s}\left(H_{\mathbf{C}}\right)$ converging to $f$ where each $f_{N}$ is a linear combination of the $u_{\alpha}$. From $(2.12)$ and $(2.15)$, $T_{h}^{F W} f_{N}$ is the stochastic extension of $T_{h}^{F H} f_{N}$. The sequence $T_{h}^{F W} f_{N}$ tends to $T_{h}^{F W} f$ in $L^{2}\left(B^{2}, \mu_{B^{2}, h}\right)$. For all complex subspace $E$ of finite dimension in $H^{2}$, one has:

$$
\left\|R_{E} T_{h}^{F H}\left(f_{N}-f\right)\right\|_{L^{2}\left(E, \mu_{E, h}\right)} \leq\left\|f_{N}-f\right\| .
$$

Consequently, in view of Theorem 2.9, the sequence of the $T_{h}^{F W} f_{N}$, stochastic extensions of the $T_{h}^{F H} f_{N}$, converges to the stochastic extension of $T_{h}^{F H} f$, which is therefore equal to $T_{h}^{F W} f$.

ii) With the above notations, the Segal Bargmann standard properties within the finite dimensional framework ([15]) show that, for all $X$ in $H^{2}$ and for any $N$ :

$$
T_{h}^{F H} f_{N}(X)=\int_{B^{2}} B_{h}(X, Y) T_{h}^{F W} f_{N}(Y) d \mu_{B^{2}, h}(Y) .
$$

From Definition (2.4), for all $X$ in $H^{2}$, the sequence $T_{h}^{F H} f_{N}(X)$ converges to $T_{h}^{F H} f(X)$. Besides, the sequence $\left(T_{h}^{F W} f_{N}\right)$ tends to $T_{h}^{F W} f$ in $L^{2}\left(B^{2}, \mu_{B^{2}, h}\right)$ and we consequently have, since the function $Y \rightarrow B_{h}(X, Y)$ belongs to $L^{2}\left(B^{2}, \mu_{B^{2}, h}\right)$ :

$$
\lim _{N \rightarrow \infty} \int_{B^{2}} B_{h}(X, Y) T_{h}^{F W}\left(f_{N}-f\right)(Y) d \mu_{B^{2}, h}(Y)=0 .
$$

One then obtains $(2.18)$.

\subsection{Segal Bargmann transform and Wick product: proof of Proposition 1.4 and Theorem 1.5}

Proof of Proposition 1.4. We consider the Hilbertian basis $\left(u_{\alpha}\right)$ of (2.1), and equality (2.21). Setting $\varphi=I(f, g)$, we have, for all multi-indices $\gamma$ :

$$
<\varphi, u_{\gamma}>=\sum_{\alpha+\beta=\gamma}<f, u_{\alpha}><g, u_{\beta}>\left[\frac{\gamma !}{\alpha ! \beta !}\right]^{1 / 2} .
$$


From Cauchy-Schwarz:

$$
\left(R^{\prime \prime}\right)^{|\gamma|}\left|<\varphi, u_{\gamma}>\right|^{2} \leq \sum_{\alpha+\beta=\gamma} R^{\alpha}\left|<f, u_{\alpha}>\right|^{2}\left(R^{\prime}\right)^{\beta}\left|<g, u_{\beta}>\right|^{2} \sum_{\alpha+\beta=\gamma} \frac{\gamma !}{\alpha ! \beta !} \frac{\left(R^{\prime \prime}\right)^{|\gamma|}}{R^{|\alpha|}\left(R^{\prime}\right)^{|\beta|}}
$$

If $\left(1 / R^{\prime \prime}\right)=(1 / R)+\left(1 / R^{\prime}\right)$ then the latter sum equals to 1 . Because $\left(u_{\alpha}\right)_{|\alpha|=N}$ is an orthonormal basis of $H^{(N)}, f$ belongs to $\mathcal{F}_{s}^{R}\left(H_{\mathbf{C}}\right)$ if and only if

$$
\|f\|_{R}^{2}=\sum\left|<f, u_{\alpha}>\right|^{2} R^{|\alpha|}<\infty
$$

Therefore, the Proposition is proved.

Proof of Theorem 1.5. Point i). We use the Hilbertian basis $\left(u_{\alpha}\right)$ of (2.1). From (2.1) and (1.3), one observes:

$$
I\left(u_{\alpha}, u_{\beta}\right)=\left[\frac{(\alpha+\beta) !}{\alpha ! \beta !}\right]^{1 / 2} u_{\alpha+\beta}
$$

for any multi-indices $\alpha$ and $\beta$. Therefore, if $f$ and $g$ belong to $\mathcal{F}_{s}^{\text {fin }}\left(H_{\mathbf{C}}\right)$ (defined in Section 1 ), one verifies that:

$$
T_{h}^{F H} I(f, g)(X)=\sum_{\alpha, \beta}<f, u_{\alpha}><g, u_{\beta}>\left[\frac{(\alpha+\beta) !}{\alpha ! \beta !}\right]^{1 / 2} T_{h}^{F H} u_{\alpha+\beta}(X) .
$$

According to Proposition 2.5:

$$
T_{h}^{F H} f(X)=\sum_{\alpha}<f, u_{\alpha}>\Phi_{\alpha, h}(X)
$$

From (2.9), one verifies:

$$
\sqrt{(\alpha+\beta) !} \Phi_{\alpha+\beta, h}(X)=\sqrt{\alpha !} \Phi_{\alpha, h}(X) \sqrt{\beta !} \Phi_{\beta, h}(X) .
$$

Point ii). One knows by Proposition 2.8 that $T_{h}^{F H} I(f, g), T_{h}^{F H} f$ and $T_{h}^{F H} g$ have the stochastic extensions $T_{h}^{F W} I(f, g), T_{h}^{F W} f$ and $T_{h}^{F W} g$ in the $L^{2}\left(B^{2}, d \mu_{B^{2}, h}\right)$ sense. Let $\left(E_{n}\right)$ be an increasing sequence of finite dimensional subspaces of $H$, with a dense union. Since $T_{h}^{F H} f \circ \tilde{\pi}_{E_{n}}$ and $T_{h}^{F H} g \circ \tilde{\pi}_{E_{n}}$ converge to $T_{h}^{F W} f$ and $T_{h}^{F W} g$ in $L^{2}\left(B^{2}, d \mu_{B^{2}, h}\right)$, the product $T_{h}^{F H} f T_{h}^{F H} g \circ \tilde{\pi}_{E_{n}}$ converges to $T_{h}^{F W} f T_{h}^{F W} g$ in $L^{1}\left(B^{2}, d \mu_{B^{2}, h}\right)$. The convergence is almost sure for a subsequence indexed by $\varphi(n)$. By point i), $\left(T_{h}^{F H} f T_{h}^{F H} g\right) \circ \tilde{\pi}_{E_{\varphi(n)}}=$ $T_{h}^{F H} I(f, g) \circ \tilde{\pi}_{E_{\varphi(n)}}$. Moreover, it converges to $T_{h}^{F W} I(f, g)$ in $L^{2}$. Extracting a further subsequence gives an almost sure convergence and the equality $T_{h}^{F W} I(f, g)=\left(T_{h}^{F W} f\right)\left(T_{h}^{F W} g\right)$ holds almost everywhere.

Proof of equality (1.10). We can write, if $X=(q, p)$ :

$$
\Psi_{X, h}=\sum_{m \geq 0} \frac{e^{-\frac{|X|^{2}}{4 h}}}{(2 h)^{m / 2} m !}\left(a^{\star}(q+i p)\right)^{m} \Psi_{0} .
$$

One checks that the coherent states belong to $\mathcal{F}_{s}^{R}\left(H_{\mathbf{C}}\right)$ for every $R \geq 1$. Let $\Psi_{X, h}^{N}$ be a truncated coherent state, the sum running on $\{m \leq N\}$ instead of $\mathbb{N}$. 
Proposition 1.4 with $R=R^{\prime}=2$ shows that $I\left(\Psi_{X, h}^{N}, \Psi_{Y, h}^{N}\right)$ converges to $I\left(\Psi_{X, h}, \Psi_{Y, h}\right)$ in $\mathcal{F}_{s}^{1}\left(H_{\mathbf{C}}\right)=$ $\mathcal{F}_{s}\left(H_{\mathbf{C}}\right)$. The computation below shows that $I\left(\Psi_{X, h}^{N}, \Psi_{Y, h}^{N}\right)=\Psi_{X+Y, h}^{N}+V_{N}$ and one easily proves that the rest $V_{N}$ converges to 0 when $N$ converges to infinity:

$$
\begin{aligned}
I\left(\Psi_{X, h}^{N}, \Psi_{Y, h}^{N}\right) & =\sum_{0 \leq m, n \leq N} \frac{e^{-\frac{|X|^{2}+|Y|^{2}}{4 h}}}{(2 h)^{m+n / 2} m ! n !} I\left(\left(a^{\star}(q+i p)\right)^{m} \Psi_{0},\left(a^{\star}\left(q^{\prime}+i p^{\prime}\right)\right)^{n} \Psi_{0}\right) \\
& =\sum_{0 \leq m, n \leq N} \frac{e^{-\frac{|X|^{2}+|Y|^{2}}{4 h}}}{(2 h)^{m+n / 2} m ! n !}\left(a^{\star}(q+i p)\right)^{m}\left(a^{\star}\left(q^{\prime}+i p^{\prime}\right)\right)^{n} \Psi_{0} \\
& =e^{-\frac{|X|^{2}+|Y|^{2}}{4 h}} \sum_{s=0}^{N} \frac{1}{s !(2 h)^{s / 2}} \sum_{m=0}^{s} \frac{s !}{m !(s-m) !}\left(a^{\star}(q+i p)\right)^{m}\left(a^{\star}\left(q^{\prime}+i p^{\prime}\right)\right)^{s-m} \Psi_{0} \\
& +e^{-\frac{|X|^{2}+|Y|^{2}}{4 h}} \sum_{s=N+1}^{2 N} \frac{1}{s !(2 h)^{s / 2}} \sum_{m=s-N}^{N} \frac{s !}{m !(s-m) !}\left(a^{\star}(q+i p)\right)^{m}\left(a^{\star}\left(q^{\prime}+i p^{\prime}\right)\right)^{s-m} \Psi_{0} \\
& =e^{-\frac{|X|^{2}+|Y|^{2}}{4 h}} \sum_{s=0}^{N} \frac{1}{s !(2 h)^{s / 2}}\left(a^{\star}\left(q+q^{\prime}+i\left(p+p^{\prime}\right)\right)\right)^{s} \Psi_{0}+V_{N}=\Psi_{X+Y, h}^{N}+V_{N} .
\end{aligned}
$$

One then deduces (1.10).

\section{Mizrahi series: proof of Theorem 1.11 .}

It suffices to prove the following equality, for all $X$ in $H^{2}$ :

$$
<B \Psi_{X h}, A^{\star} \Psi_{X h}>=\sum \frac{h^{|\alpha|}}{2^{|\alpha|} \alpha !}\left(\partial_{q}-i \partial_{p}\right)^{\alpha} \sigma_{h}^{w i c k}(A)(X)\left(\partial_{q}+i \partial_{p}\right)^{\alpha} \sigma_{h}^{w i c k}(B)(X)
$$

Proof of (3.1) for $X=0$. By Proposition 2.7, we have:

$$
<B \Psi_{0 h}, A^{\star} \Psi_{0 h}>=\sum<T_{h}^{F W} B \Psi_{0 h}, \widetilde{\Phi}_{\alpha, h}><\widetilde{\Phi}_{\alpha, h}, T_{h}^{F W} A^{\star} \Psi_{0 h}>.
$$

Applying Proposition 2.8 to $B \Psi_{0 h}$, we obtain, for $X$ in $H^{2}$ :

$$
T_{h}^{F H}\left(B \Psi_{0 h}\right)(X)=\int_{B^{2}} \mathcal{B}_{h}(X, Y) T_{h}^{F W}\left(B \Psi_{0 h}\right)(Y) d \mu_{B^{2}, h}(Y) .
$$

Differentiating the integral,

$$
\left(\partial_{x_{j}}+i \partial_{\xi_{j}}\right) T_{h}^{F H}\left(B \Psi_{0 h}\right)(X)=h^{-1} \int_{B^{2}} \ell_{e_{j}}(y+i \eta) \mathcal{B}_{h}(X, Y) T_{h}^{F W}\left(B \Psi_{0 h}\right)(Y) d \mu_{B^{2}, h}(Y) .
$$

Iterating and then setting $X=0$, one obtains

$$
\left(\partial_{x}+i \partial_{\xi}\right)^{\alpha} T_{h}^{F H}\left(B \Psi_{0 h}\right)(0)=h^{-|\alpha|} \int_{B^{2}}\left[\prod\left(\ell_{e_{j}}(y)+i \ell_{e_{j}}(\eta)\right)^{\alpha_{j}}\right] T_{h}^{F W}\left(B \Psi_{0 h}\right)(Y) d \mu_{B^{2}, h}(Y) .
$$


Equivalently,

$$
<T_{h}^{F W}\left(B \Psi_{0 h}\right), \widetilde{\Phi}_{\alpha, h}>=\left(\frac{h}{2}\right)^{|\alpha| / 2}(\alpha !)^{-1 / 2}\left(\partial_{x}+i \partial_{\xi}\right)^{\alpha} T_{h}^{F H}\left(B \Psi_{0 h}\right)(0)
$$

and the same equality holds for $T_{h}^{F W}\left(A^{\star} \Psi_{0 h}\right)$. Consequently,

$$
<B \Psi_{0 h}, A^{\star} \Psi_{0 h}>=\sum\left(\frac{h}{2}\right)^{|\alpha|}(\alpha !)^{-1}\left(\partial_{x}+i \partial_{\xi}\right)^{\alpha} T_{h}^{F H}\left(B \Psi_{0 h}\right)(0) \overline{\left(\partial_{x}+i \partial_{\xi}\right)^{\alpha} T_{h}^{F H}\left(A^{\star} \Psi_{0 h}\right)(0)} .
$$

For any bounded operator $B$ in $\mathcal{F}_{s}\left(H_{\mathbf{C}}\right)$, for all $X$ and $Y$ in $H^{2}$, set:

$$
\left(S_{h} B\right)(X, Y)=\frac{<B \Psi_{X h}, \Psi_{Y h}>}{<\Psi_{X h}, \Psi_{Y h}>} .
$$

By (2.5) and (2.4), we have:

$$
\left(S_{h} B\right)(X, Y)=e^{\frac{|X|^{2}}{4 h}} e^{-\frac{1}{2 h}(x+i \xi) \cdot(y-i \eta)}\left(T_{h}^{F H}\left(B \Psi_{X h}\right)\right)(Y) .
$$

Therefore, when $Y=(y, \eta)$ is identified with $y+i \eta$, the function $Y \rightarrow\left(S_{h} B\right)(X, Y)$ is Gateaux antiholomorphic. Similarly, the function $X \rightarrow\left(S_{h} B\right)(X, Y)$ is Gateaux holomorphic. Since the restriction of this function to the diagonal is the Wick symbol of $B$, one has,

$$
\left(\partial_{q}+i \partial_{p}\right)^{\alpha} T_{h}^{F H}\left(B \Psi_{0 h}\right)(0)=\left(\partial_{q}+i \partial_{p}\right)^{\alpha} \sigma_{h}^{w i c k}(B)(0) .
$$

The equality (3.1) is then proved for $X=0$.

Proof of (3.1) for arbitrary $X$. The coherent states $\Psi_{X, h}$ are defined in (1.15). We know that, for all $U$ and $V$ in $H^{2}$ :

$$
e^{i \Phi_{S}(U)} e^{i \Phi_{S}(V)}=e^{\frac{i}{2} \sigma(U, V)} e^{i \Phi_{S}(U+V)}
$$

where $\sigma$ is the symplectic form $\sigma((a, b),(q, p))=b \cdot q-a \cdot p$. Using (1.15) and (3.3), we obtain:

$$
\sigma_{h}^{w i c k}\left(V_{h}(X) A V_{h}(-X)\right)(Y)=\left(\sigma_{h}^{w i c k}(A)\right)(Y-X) \text {. }
$$

One then deduces equality (3.1) for any arbitrary $X$ and Theorem 1.11 is proved.

\section{Weyl symbol composition: proof of Theorem 1.12 .}

Proof of points i) and ii) of Theorem 1.12. According to (1.24), we have, choosing a Hilbertian basis $\left(e_{j}\right)$ :

$$
\begin{gathered}
\left\|\sigma\left(\nabla_{1}, \nabla_{2}\right)^{k}(F \otimes G)\right\|_{\infty} \leq\|F\|_{Q}\|G\|_{Q}\left(\sum_{j} 2 Q\left(e_{j}, 0\right)^{1 / 2} Q\left(0, e_{j}\right)^{1 / 2}\right)^{k} \\
\leq\|F\|_{Q}\|G\|_{Q}\left(\sum_{j}\left(Q\left(e_{j}, 0\right)+Q\left(0, e_{j}\right)\right)\right)^{k}
\end{gathered}
$$




$$
\leq\|F\|_{Q}\|G\|_{Q}\left(\operatorname{Tr} A_{Q}\right)^{k}
$$

From (1.25), one sees:

$$
\left\|C_{k}^{\text {weyl }}(F, G)\right\|_{\infty} \leq \frac{1}{2^{k} k !}\left\|\sigma\left(\nabla_{1}, \nabla_{2}\right)^{k}(F \otimes G)\right\|_{\infty} .
$$

We estimate similarly the derivatives and we deduce $(1.26)$. The series defining $K_{h}^{\text {weyl }}(F, G)$ in Theorem 1.12 is then convergent and the asymptotic expansion iii) is valid.

Proof of point iii) Equality (1.28) comes from the next Lemma. Indeed, this Lemma shows that the operators in the left and right hand sides of (1.28) share the same Wick symbol. Thus they are equal.

Lemma 4.1. For all $F$ and $G$ in $S\left(H^{2}, Q_{A}\right)$, one has:

$$
\sum_{k=0}^{\infty} h^{k} C_{k}^{\text {wick }}\left(H_{h / 2} F, H_{h / 2} G\right)=H_{h / 2}\left[\sum_{k=0}^{\infty} h^{k} C_{k}^{w e y l}(F, G)\right]
$$

where $C_{k}^{\text {weyl }}(F, G)$ is defined in (1.25).

Proof of the Lemma. We denote by $\Phi(h)$ the right hand side and by $\Psi(h)$ the left hand side of (4.1). For every function $F$ belonging to $S\left(H^{2}, Q\right)$, with $A_{Q}$ trace class, the heat operator can also be written as:

$$
H_{h / 2} F=\sum_{k=0}^{\infty} \frac{(h / 4)^{k}}{k !} \Delta^{k} F .
$$

Since $F$ belongs to $S\left(H^{2}, Q\right)$, then we have (4.2), where the series is convergent and defines an element of $S\left(H^{2}, Q\right)$. Looking at the powers of $h$, we are led to compare the coefficient of $h^{k}$ in $\Phi(h)$

$$
a_{k}=\sum_{s+\ell=k} \frac{1}{4^{\ell} \ell !} \Delta^{\ell} C_{s}^{w e y l}(F, G)
$$

with the coefficient of $h^{k}$ in $\Psi(h)$

$$
b_{k}=\sum_{s+\ell+u=k} \frac{1}{4^{\ell+s} s ! \ell !} C_{u}^{w i c k}\left(\Delta^{\ell} F, \Delta^{s} G\right) .
$$

Let $\sigma\left(\nabla_{1}, \nabla_{2}\right)$ be the differential operator on $H^{2} \times H^{2}$ defined in (1.24). We denote by $R$ the restriction operator defined for all functions $F$ on $H^{2} \times H^{2}$ by:

$$
(R F)(X)=F(X, X)
$$

We have

$$
\Delta R=R \widetilde{\Delta}, \quad \widetilde{\Delta}=\Delta_{1}+\Delta_{2}+2 \nabla_{1} \cdot \nabla_{2}, \quad \nabla_{1} \cdot \nabla_{2}=\sum_{j} \frac{\partial^{2}}{\partial x_{j} \partial y_{j}}+\frac{\partial^{2}}{\partial \xi_{j} \partial \eta_{j}} .
$$

We then have to compare the following coefficients (operators acting on functions defined on $H^{2} \times H^{2}$ )

$$
A_{k}=\sum_{s+\ell=k} \frac{1}{(2 i)^{s} 4^{\ell} s ! \ell !}(\widetilde{\Delta})^{\ell}\left(\sigma\left(\nabla_{1}, \nabla_{2}\right)\right)^{s}
$$




$$
B_{k}=\sum_{s+\ell+n=k} \frac{1}{2^{n} 4^{\ell+s} s ! \ell !} \sum_{|\alpha|=n} \frac{1}{\alpha !}\left(\partial_{x}-i \partial_{\xi}\right)^{\alpha}\left(\partial_{y}+i \partial_{\eta}\right)^{\alpha} \Delta_{1}^{\ell} \Delta_{2}^{s} .
$$

Expanding $(\widetilde{\Delta})^{\ell}$ using the binomial formula, we are led to compare:

$$
\begin{gathered}
A_{k}^{\prime}=\sum_{\ell=0}^{k} \sum_{u+s \leq \ell} \frac{i^{\ell-k}}{2^{k+u+s}(k-\ell) ! u ! s !(\ell-u-s) !}\left(\nabla_{1} \cdot \nabla_{2}\right)^{\ell-u-s}\left(\sigma\left(\nabla_{1}, \nabla_{2}\right)\right)^{k-\ell} \Delta_{1}^{u} \Delta_{2}^{s} \\
B_{k}^{\prime}=\sum_{s+u+|\alpha|=k} \frac{1}{2^{k+u+s} u ! s ! \alpha !}\left(\partial_{x}-i \partial_{\xi}\right)^{\alpha}\left(\partial_{y}+i \partial_{\eta}\right)^{\alpha} \Delta_{1}^{u} \Delta_{2}^{s} .
\end{gathered}
$$

Thus, we have to compare, for $t=u+s$ being integers not greater than $k$ :

$$
\begin{gathered}
A_{k}(t)=\sum_{\ell=t}^{k} \frac{i^{\ell-k}}{(k-\ell) !(\ell-t) !}\left(\nabla_{1} \cdot \nabla_{2}\right)^{\ell-t}\left(\sigma\left(\nabla_{1}, \nabla_{2}\right)\right)^{k-\ell} \\
B_{k}(t)=\sum_{|\alpha|=k-t} \frac{1}{\alpha !}\left(\partial_{x}-i \partial_{\xi}\right)^{\alpha}\left(\partial_{y}+i \partial_{\eta}\right)^{\alpha} .
\end{gathered}
$$

These terms are the same. Indeed, call

$$
\chi\left(\begin{array}{c}
\vdots \\
\chi_{n} \\
\vdots
\end{array}\right)=\left(\begin{array}{c}
\vdots \\
\left(\partial_{x_{n}}-i \partial_{\xi_{n}}\right)\left(\partial_{y_{n}}+i \partial_{\eta_{n}}\right) \\
\vdots
\end{array}\right)=\left(\begin{array}{c}
\vdots \\
\partial_{x_{n}} \partial_{y_{n}}+\partial_{\xi_{n}} \partial_{\eta_{n}}-i\left(\partial_{\xi_{n}} \partial_{y_{n}}-\partial_{x_{n}} \partial_{\eta_{n}}\right) \\
\vdots
\end{array}\right) .
$$

The term $B_{k}(t)$ is nothing but $\frac{1}{(k-t) !}\left(\sum_{n} \chi_{n}\right)^{k-t}$ expanded with the complex differentiations, while

$$
A_{k}(t)=\sum_{\ell^{\prime}=0}^{k-t} \frac{i^{\ell^{\prime}}}{\left(k-t-\ell^{\prime}\right) !\left(\ell^{\prime}\right) !}\left(\nabla_{1} \cdot \nabla_{2}\right)^{\ell^{\prime}}\left(\sigma\left(\nabla_{1}, \nabla_{2}\right)\right)^{k-t-\ell^{\prime}}
$$

is obtained by separating real and imaginary part.

\section{References}

[1] S. Albeverio,Y. G. Kondratiev, and M. Röckner, Analysis and geometry on configuration spaces, J. Funct. Anal., 154, 1998.

[2] Z. Ammari, F. Nier, Mean field limit for bosons and infinite dimensional phase-space analysis, Ann. Henri Poincaré, 9,2008,8, 1503-1574.

[3] L. Amour, L. Jager, J. Nourrigat, On bounded pseudodifferential operators in Wiener spaces, J. Funct. Anal. 269 (2015), no. 9, 2747-2812.

[4] L. Amour, L. Jager, J. Nourrigat, Infinite dimensional semiclassical analysis and applications to a model in NMR, J. Math. Phys., to be published. 
[5] L. Amour, R. Lascar, J. Nourrigat, Weyl calculus in Wiener spaces and in QED, J. Pseudo-Differ. Oper. Appl., 1 (2019), 1-47.

[6] L. Amour, R. Lascar, J. Nourrigat, Weyl calculus in QED I. The unitary group., J. Math. Phys., 58 (2017)

[7] D. M. Appleby, Husimi transform of an operator product, J. Phys. A, 33, 2000, 21, 3903-3915.

[8] J. Baez, I. E. Segal, Z-F Zhou, Introduction to algebraic and constructive quantum field theory, Princeton Series in Physics. Princeton University Press, Princeton, NJ, 1992.

[9] Berezansky, Y. G. Kondratiev, Spectral methods in infinite-dimensional analysis. Vol. 1, Translated from the 1988 Russian original by P. V. Malyshev and D. V. Malyshev. Mathematical Physics and Applied Mathematics, 12/2. Kluwer Academic Publishers, Dordrecht, 1995.

[10] F. A. Berezin, The method of second quantization, Translated from the Russian, Pure and Applied Physics, Vol. 24 Academic Press, New York-London 1966.

[11] F. Bloch, Nuclear Induction, Physical Review 70 460-473, (1946).

[12] R. H. Cameron, W. T. Martin, Transformations of Wiener integrals under translations, Ann. of Math. (2), 45, 1944.

[13] J. Dereziński, C. Gérard, Asymptotic completeness in quantum field theory. Massive Pauli-Fierz Hamiltonians. Rev. Math. Phys. 11 (1999), no. 4, 383-450.

[14] B. K. Driver and B. C. Hall, Yang-Mills theory and the Segal-Bargmann transform, Comm. Math. Phys., 201, 1999, 2, 249-290

[15] G. B. Folland, Harmonic analysis in phase space. Annals of Mathematics Studies, 122. Princeton University Press, Princeton, NJ, 1989.

[16] C. Gérard, On the Existence of Ground States for Massless Pauli-Fierz Hamiltonians, Ann. Henri Poincaré, 1 (2000) 443-459.

[17] J. Glimm, Mathematical problems in the foundations of quantum field theory, Lectures in Modern Analysis and Applications. II, 58-67, Lecture Notes in Mathematics, Vol. 140. Springer, Berlin, 1970.

[18] J. Glimm, A. Jaffe, Quantum field theory and statistical mechanics, Birkhäuser Boston, Inc., Boston, MA, 1985.

[19] L. Gross, Measurable functions on Hilbert space, Trans. Amer. Math. Soc. 105 (1962) 372-390.

[20] L. Gross, Abstract Wiener spaces, Proc. 5th Berkeley Sym. Math. Stat. Prob, 2, (1965), 31-42.

[21] L. Gross, Abstract Wiener measure and infinite dimensional potential theory, in Lectures in modern Analysis and applications, II, Lecture Notes in Math 140, 84-116, Springer (1970).

[22] L. Gross, Potential theory on Hilbert space, J. Functional Analysis 1, (1967) 123-181.

[23] B. C. Hall, The heat operator in infinite dimensions, Infinite dimensional stochastic analysis, QP-PQ: Quantum Probab. White Noise Anal., 22, 161-174, World Sci. Publ., Hackensack, NJ, 2008. 
[24] B. C. Hall, Holomorphic methods in analysis and mathematical physics, First Summer School in Analysis and Mathematical Physics (Cuernavaca Morelos, 199, Contemp. Math.,260, 1-59, Amer. Math. Soc., Providence, RI, 2000.

[25] T. Hida, Brownian motion, Applications of Mathematics, 11, Springer-Verlag, New York-Berlin, 1980.

[26] T. Hida, Causal analysis in terms of white noise, Quantum fields-algebras, processes (Proc. Sympos., Univ. Bielefeld, Bielefeld, 1978), 1-19, Springer, Vienna.

[27] F. Hiroshima, Multiplicity of ground states in quantum field models: applications of asymptotic fields, J. Funct. Anal. 224 (2005), no. 2, 431-470.

[28] L. Hörmander, The analysis of linear partial differential operators, Volume III, Springer, 1985.

[29] M. Hübner, H. Spohn, Radiative decay: nonperturbative approaches, Rev. Math. Phys. 7 (1995), no. 3, 363-387.

[30] K. Itô, Multiple Wiener integral, J. Math. Soc. Japan, 3, 1951, 157-169.

[31] L. Jager, Stochastic extensions of symbols in Wiener spaces and heat operator, arXiv:1607.02253, july 2016 .

[32] S. Janson, Gaussian Hilbert spaces, Cambridge Tracts in Maths, 129, Cambridge Univ. Press (1997).

[33] P. Krée, R. Rączka, Kernels and symbols of operators in quantum field theory, Annales de l'I.H.P. Section A, 28, 1, (1978), 48-73.

[34] I. Kubo, S. Takenaka, Calculus on Gaussian white noise. I, Proc. Japan Acad. Ser. A Math. Sci., 56, 1980, 8, 376-380.

[35] I. Kubo, S. Takenaka, Calculus on Gaussian white noise. II, Proc. Japan Acad. Ser. A Math. Sci., $56,1980,9,411-416$.

[36] H. H. Kuo, Gaussian measures in Banach spaces. Lecture Notes in Mathematics, Vol. 463. Springer, Berlin-New York, 1975.

[37] H. H. Kuo, Introduction to stochastic integration, Universitext, Springer, New York, 2006

[38] B. Lascar, Une classe d'opérateurs elliptiques du second ordre sur un espace de Hilbert, J. Funct. Anal. 35 (1980), no. 3, 316-343.

[39] P. Malliavin, Stochastic analysis, Grundlehren der Mathematischen Wissenschaften 313, SpringerVerlag, Berlin, 1997.

[40] P.-A. Meyer, Distributions, noyaux, symboles d'après Krée, Séminaire de Probabilités, XXII, Lecture Notes in Math., 1321, 467-476, Springer, Berlin, 1988.

[41] P.-A. Meyer, Quantum probability for probabilists, Lecture Notes in Mathematics, 1538, SpringerVerlag, Berlin, 1993.

[42] P.-A. Meyer, J. A. Yan, À propos des distributions sur l'espace de Wiener, Séminaire de Probabilités, XXI, Lecture Notes in Math., 1247, 8-26, Springer, Berlin, 1987 
[43] M. M. Mizrahi, On the semiclassical expansion in quantum mechanics for arbitrary Hamiltonians, J. Math. Phys. (1977), 18, 786-790, 34.

[44] D. Nualart, The Malliavin calculus and related topics, Probability and its Applications (New York), Second edition, 2006.

[45] M. Reed, B. Simon, Methods of modern mathematical physics, Vol. I, Functional analysis, Academic Press, 1975.

[46] M. Reed, B. Simon, Methods of modern mathematical physics, Vol. II, Fourier Analysis, selfadjointness, Academic Press, 1975.

[47] F. A. Reuse, Electrodynamique et Optique Quantiques, Presses Polytechniques et Universitaires Romandes, Lausanne, 2007.

[48] I. Segal, Tensor algebras over Hilbert spaces, I, Trans. Amer. Math. Soc, 81 (1956), 104-134.

[49] D. Shale. Linear symmetries of free boson fields. Trans. Amer. Math. Soc., 103, 149-167, 1962.

[50] B. Simon, The $P(\Phi)_{2}$ Euclidean (Quantum) Field theory, Princeton Series in Physics, Princeton Univ. Press, Princeton (1974).

[51] B. Simon, Functional integration and quantum physics, Pure and Applied Mathematics, 86, Academic Press, Inc. [Harcourt Brace Jovanovich, Publishers], New York-London, 1979.

[52] V. A. Skorohod, Integration in Hilbert space, Translated from the Russian by Kenneth Wickwire, Ergebnisse der Mathematik und ihrer Grenzgebiete, Band 79, Springer-Verlag, New York-Heidelberg, 1974.

[53] H. Spohn, Ground state(s) of the spin-boson Hamiltonian, Comm. Math. Phys, 123 277-304, (1989).

[54] H. Spohn, Dynamics of charged particles and their radiation field, Cambridge University Press, Cambridge, 2004.

[55] A. Unterberger, Les opérateurs métadifférentiels, in Complex analysis, microlocal calculus and relativistic quantum theory, Lecture Notes in Physics 126 (1980) 205-241.

[56] N. Wiener, The Homogeneous Chaos, Amer. J. Math., 60, 1938, 4, 897-936.

laurent.amour@univ-reims.fr

LMR CNRS FRE 2011, Université de Reims Champagne-Ardenne, Moulin de la Housse, BP 1039, 51687 REIMS Cedex 2, France.

lisette.jager@univ-reims.fr

LMR CNRS FRE 2011, Université de Reims Champagne-Ardenne, Moulin de la Housse, BP 1039, 51687 REIMS Cedex 2, France.

jean.nourrigat@univ-reims.fr

LMR CNRS FRE 2011, Université de Reims Champagne-Ardenne, Moulin de la Housse, BP 1039, 51687 REIMS Cedex 2, France. 